Kelson James Silva de Almeida

\title{
Ultrassonografia transcraniana combinada a teste de olfação comparados à imagem molecular com TRODAT para diagnóstico da doença de Parkinson
}

Tese apresentada à Faculdade de Medicina da Universidade de São Paulo para obtenção do Título de Doutor em Ciências

Programa de Neurologia

Orientador: Prof. Dr. Edson Bor-Seng-Shu

São Paulo 
Kelson James Silva de Almeida

\title{
Ultrassonografia transcraniana combinada a teste de olfação comparados à imagem molecular com TRODAT para diagnóstico da doença de Parkinson
}

\author{
Tese apresentada à Faculdade de \\ Medicina da Universidade de São Paulo \\ para obtenção do Título de Doutor em \\ Ciências \\ Programa de Neurologia \\ Orientador: Prof. Dr. Edson Bor-Seng-Shu
}

São Paulo 
Dados Internacionais de Catalogação na Publicação (CIP)

Preparada pela Biblioteca da

Faculdade de Medicina da Universidade de São Paulo

Creprodução autorizada pelo autor

Almeida, Kelson James Silva de

Ultrassonografia transcraniana combinada a teste de olfação comparados à imagem molecular com TRODAT para diagnóstico da doença de Parkinson / Kelson James Silva de Almeida. -- São Paulo, 2016

Tese(doutorado)--Faculdade de Medicina da Universidade de São Paulo. Programa de Neurologia.

Orientador: Edson Bor-Seng-Shu.

Descritores: 1.Doença de Parkinson 2.Substância negra 3.Ultrassonografia 4.Olfato 5.Tomografia computadorizada de emissão de fóton único 6.Transtornos do olfato

USP/FM/DBD-328/16 


\section{DEDICATÓRIA}

Ao professor Edson Bor-Seng-Shu, meu exemplo de homem e de médico, a quem dedico os resultados e as boas intenções da minha jornada. Subsidium datum est levare cor. Subsidium datum est mark mens.

A Deus, pela proteção e pelas oportunidades amparadas de paz.

À minha mãe Cassandra, minha fonte de sustentação, pela dedicação, resignação, obstinação e carinho. Agradeço seu amor infinito.

À minha avó Inês, exemplo de retidão, pela presença, preocupação e amor inocente.

Ao meu pai Antônio, pela vontade indelével de sempre cooperar e se fazer presente.

À minha irmã Jaíra, pela alegria e capacidade de enfrentar os obstáculos da vida.

Às tias Hilda, Kátia, Sílvia e aos primos, pela torcida e estima incomparáveis.

Aos que me apoiam, pela admiração, presença e oportunidades. Agradeço os ensinamentos que me ajudam a caminhar pelo mundo.

Aos meus pacientes e alunos, fontes de aprendizagem e crescimento. 


\section{AGRADECIMENTOS}

Ao Prof. Edson Bor-Seng-Shu, meu segundo pai na vida, exemplo de motivação, justiça e honestidade. Pela capacidade de aglutinar alunos e prover o crescimento deles. Pelo intelecto aguçado em raciocínio clínico e pesquisa médica. Agradeço sua confiança e a oportunidade de aprender sobre a vida; por suas palavras, compreensão e ombro amigo nos momentos difíceis.

Ao Prof. Marcelo de Lima Oliveira, fonte incomensurável de conhecimentos; pela paciência, humildade e espírito docente.

Ao Prof. Egberto Reis Barbosa, pelos ensinamentos sólidos na área dos anormalidades do movimento. Pelo apoio, disponibilidade mais que profissional.

Ao Prof. Henrique Ballalai Ferraz, grande neurologista e cientista na subespecialidade de anormalidades do movimento, pela colaboração científica e oportunidade de realizar esta tese. À equipe da Universidade Federal de São Paulo pela parceria em disponibilizar pacientes para o laboratório de Neurossonologia do HCFMUSP, em especial Drs. José Luiz Pedroso, André Felício e Orlando Barsottini.

Aos Profs. Raimundo Nonato Campos-Sousa e Raimundo Pereira da SilvaNeto, pela sustentação durante minha graduação e o esforço em mostrar-me uma especialidade de futuro.

Aos Profs. Manoel Jacobsen Teixeira e Ricardo Nitrini, por moldarem a formação dos profissionais da nossa escola de Neurociências. 
NORMALIZAÇÃO ADOTADA

Esta tese está de acordo com as seguintes normas, em vigor no momento desta publicação:

Referências: adaptado de International Committee of Medical Journals Editors (Vancouver).

Universidade de São Paulo. Faculdade de Medicina. Divisão de Biblioteca e Documentação. Guia de apresentação de dissertações, teses e monografias. Elaborado por Anneliese Carneiro da Cunha, Maria Julia de A. L. Freddi, Maria F. Crestana, Marinalva de Souza Aragão, Suely Campos Cardoso, Valéria Vilhena. 3a ed. São Paulo: Divisão de Biblioteca e Documentação; 2011.

Abreviaturas dos títulos dos periódicos de acordo com List of Journals Indexed in Index Medicus. 


\section{SUMÁRIO}

Lista de abreviaturas

Lista de anexos

Resumo

Summary

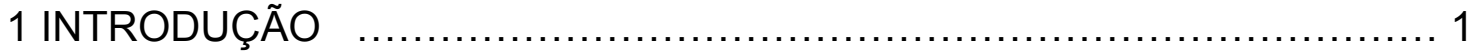

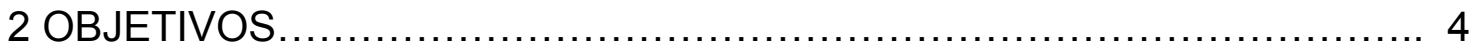

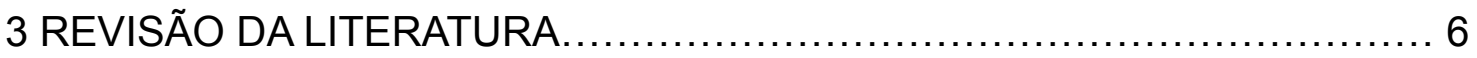

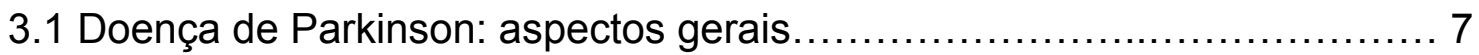

3.2 Ultrassonografia transcraniana na doença de Parkinson ....................10

3.3 Estudos transversais com ultrassonografia transcraniana $\ldots \ldots \ldots \ldots \ldots \ldots \ldots$

3.4 Estudos longitudinais com ultrassonografia transcraniana $\ldots \ldots \ldots \ldots \ldots \ldots .12$

3.5 Imagem molecular na doença de Parkinson........................ 14

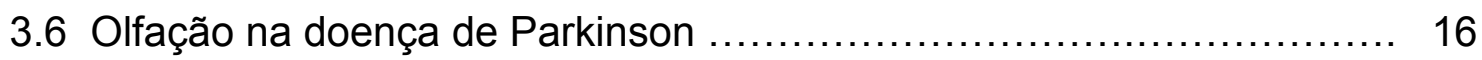

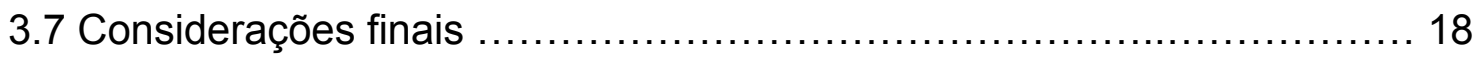

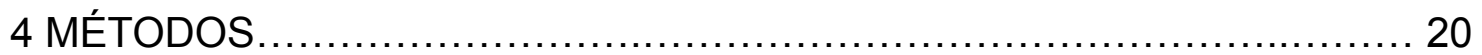

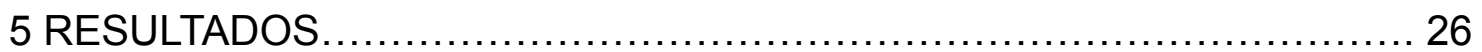

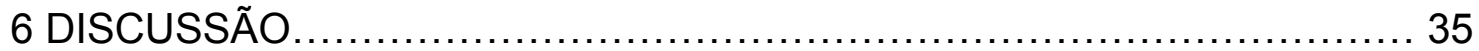

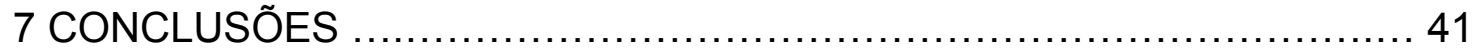

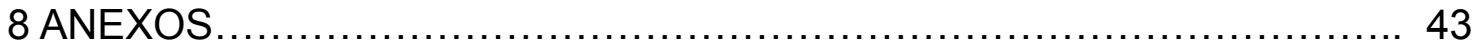

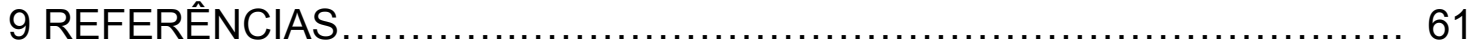

Apêndices 


\section{LISTA DE ABREVIATURAS}

${ }^{11} \mathrm{C}$

Carbono

${ }^{11} \mathrm{C}-\mathrm{NMSP} \quad{ }^{11} \mathrm{C}-\mathrm{N}$-metilespiperona

${ }^{18} \mathrm{~F}$-DMFP $\quad{ }^{18} \mathrm{~F}$-desmetoxifaliprida

${ }^{18} \mathrm{~F} \quad$ Flúor

123 I lodo

99mTc Tecnécio

${ }^{18}$ F-DOPA Fluorodopa

AMS Atrofia de múltiplos sistemas

DCB Degeneração córticobasal

DCL Demência associada a corpos de Lewy

DP Doença de Parkinson

HCFMUSP Hospital das Clínicas da Faculdade de Medicina da Universidade de São Paulo

HIAE Hospital Israelita Albert Einstein

HY Escala de Hoehn e Yahr modificada

HSN Hiperecogenicidade em região da substância negra

MMEE Miniexame do estado mental

PET Tomografia por emissão de pósitrons

PSP Paralisia supranuclear progressiva

TCSREM Transtorno comportamental do sono REM

TAD Transportadores de dopamina

TE

Tremor essencial 
TRODAT Tropane Dopamine Transporter

ROC Receiver Operating Characteristic

ROI Regiões de interesse

RM Ressonância nuclear magnética

SPA Síndromes parkinsonianas atípicas

SN Substância negra

SPECT Single-photon emission computed tomography

SST-16 Teste de olfação Sniffin' Sticks de 16 ítens

UNIFESP Universidade Federal de São Paulo

UPDRS Unified Rating Parkinson`s Disease Rating Scale

USTC Ultrassonografia transcraniana

SE Escala de Schwab e England

aSYN Alfa sinucleína 


\section{LISTA DE ANEXOS}

Anexo A - "United Kingdom Parkinson`s Disease Society Brain Bank Clinical Diagnostic Criteria"

Anexo B - "Unified Rating Parkinson`s Disease Rating Scale" - Parte III

Anexo C - Escala de Hoenh and Yahr modificada

Anexo D - Escala de Schwab and England

Anexo E - Miniexame do Estado Mental

Anexo F - Inventário de depressão de Beck

Anexo G - Comprovante de aprovação no Comitê de ética em pesquisa 


\section{RESUMO}

ALMEIDA, K.J.S. Ultrassonografia transcraniana combinada a teste de olfação comparados à imagem molecular com TRODAT para diagnóstico da doença de Parkinson [tese]. São Paulo: Faculdade de Medicina, Universidade de São Paulo; 2016.

INTRODUÇÃO: O diagnóstico da doença de Parkinson (DP) pode ser um desafio, principalmente nas fases precoces da doença. O diagnóstico acurado desta condição requer mais que a avaliação clínica isolada. A Tomografia computadorizada do crânio de fóton único (SPECT) e a ultrassonografia transcraniana (USTC) podem ser úteis na diferenciação entre a DP e as síndromes parkinsonianas atípicas ou entre a DP e o tremor essencial. 0 presente estudo objetivou investigar a acurácia da USTC combinada com o teste de olfação Sniffin' Sticks (SST-16) para diferenciar pacientes com DP de controles saudáveis e comparar com a acurácia do SPECT com 99mTcTRODAT-1 (TRODAT). MÉTODOS: Trata-se de um estudo transversal que incluiu pacientes com DP segundo critérios do United Kingdom Parkinson's disease Society e um grupo controle de indivíduos saudáveis pareados para idade e gênero. Os pacientes foram examinados por um especialista em distúrbios do movimento e submetidos a SPECT encefálico com TRODAT, USTC e SST-16. Curvas Receiver Operating Characteristic (ROC) foram obtidas para definir os pontos de corte dos métodos avaliados para detecção de DP. RESULTADOS: Vinte indivíduos com DP (13 homens e 7 mulheres) e 9 participantes saudáveis foram admitidos no estudo. A idade mediana de início dos sintomas foi de 56,5 anos e a mediana do tempo de duração da doença foi de 5 anos. Maior área de ecogênica da substância negra (SN) foi observada no grupo com DP ( $p=0,013)$. Área ecogênica da $S N$ de $0,22 \mathrm{~cm}^{2}$ foi definida pela curva ROC para detecção de DP, com acurácia de 79\%. O ponto de corte do potencial de ligação do TRODAT no striatum foi 0,90 , com acurácia de $99 \%$ para o diagnóstico de DP. Escore do SST-16 maior ou igual a 10 pontos foi o ponto de corte para detecção de DP, com acurácia de 85,8\%. A combinação da USTC com teste da olfação levou à acurácia de 95\% para detecção de DP. CONCLUSÃO: A combinação da USTC com SST-16 eleva a capacidade de 
detecção da DP. A acurácia da USTC combinada ao SST-16 para identificar pacientes com DP idiopática aproximou-se da acurácia do SPECT com TRODAT.

Descritores: doença de Parkinson; Substância negra; Ultrassonografia; Olfato; Tomografia computadorizada de emissão de fóton-único; Transtornos do olfato. 


\section{SUMMARY}

ALMEIDA, K.J.S. Combined assessment by transcranial sonography and Sniffin' Sticks test compared to brain TRODAT SPECT for Parkinson's disease diagnosis. [Thesis]. São Paulo: "Faculdade de Medicina, Universidade de São Paulo"; 2016.

INTRODUCTION: Diagnosing Parkinson's disease (PD) can be challenging, especially in the early stages of the disease. An accurate diagnosis requires more than clinical findings alone. Brain single-photon emission computed tomography (SPECT) and transcranial sonography (TCS) are helpful for diagnosing PD and differentiating it from atypical parkinsonian syndromes as well as essential tremor. This study aimed to investigate the accuracy of TCS combined with the Sniffin' sticks olfactory test (SST-16) for differentiation between idiopathic PD patients and healthy controls compared to that of ${ }^{99 \mathrm{mT}} \mathrm{Tc}-$ TRODAT-1 SPECT (TRODAT). METHODS: A cross-sectional study included PD patients diagnosed in accordance with United Kingdom PD Society Brain Bank criteria and a control group of age and sex-matched healthy subjects. All patients were examined by a movement disorder specialist and underwent brain SPECT using TRODAT, TCS examination and SST-16 test. Receiver Operating Characteristic (ROC) curves were used to calculate cut-off points for TCS, Striatal TRODAT binding potentials and SST-16. The area under the ROC curve determined the accuracy of the method. RESULTS: Twenty patients with PD (13 males and 7 females) and nine healthy subjects were included. Median age of PD onset was 56.5 years with median disease duration of 5 years. A larger substantia nigra (SN) echogenic area was observed in the PD group $(p=0.013)$. $\mathrm{SN}$ echogenic area cut-off point of $0.22 \mathrm{~cm}^{2}$ was obtained from a ROC curve for PD diagnosis. Considering this cut-off point, TCS accuracy was estimated at $79.2 \%$ for PD diagnosis. The cut-off value of 0.90 for striatal TRODAT binding was associated with $99 \%$ accuracy for the diagnosis of PD. SST-16 values equal or greater than 10 points showed a $85.8 \%$ accuracy for PD diagnosis. Combination of both SST-16 and TCS improved the accuracy to $95 \%$ for PD diagnosis. CONCLUSION: Combined assessment of SST-16 and TCS are reliable and highly accurate for distinguishing PD patients from healthy controls. 
The accuracy of TCS combined with SST-16 for differentiation between idiopathic PD patients and healthy controls is similar to that of SPECT TRODAT.

Descriptors: Parkinson disease; Substantia nigra; Ultrasonography; Smell; Tomography, Emission-Computed, Single-Photon; Olfaction disorders. 
INTRODUÇÃO 
O diagnóstico da doença de Parkinson (DP) é essencialmente clínico e baseado nos critérios do United Kingdom Parkinson’s Disease Society (Hughes et al., 1992). Em condições clinicas como no tremor essencial (TE) e nas síndromes parkinsonianas atípicas (SPA), o diagnóstico diferencial com a DP pode ser desafiador, principalmente nas fases precoces dessas doenças (Doepp et al., 2008; Hughes et al., 2002). Nesse contexto, métodos complementares podem prover marcadores diagnósticos para DP como a tomografia por emissão de fóton único (do inglês, single-photon emission computerized tomography ou SPECT), tomografia por emissão de pósitrons (do inglês, position emission tomography ou PET), ultrassonografia transcraniana (USTC) e testes do olfato.

Para aquisição da imagem dos transportadores de dopamina (TDA), utiliza-se as técnicas SPECT ou PET usando radioligantes com alta afinidade a essas moléculas. A técnica SPECT com 99mTc-TRODAT-1, do inglês, tropane dopamine transporter, apresenta acurácia satisfatória para identificar déficit dopaminérgico na via nigroestriatal, presente nos parkinsonismos degenerativos. A diminuição da quantidade de TDA no striatum reflete degeneração dos neurônios dopaminérgicos da substância negra (SN) e pode indicar o diagnóstico e a fase evolutiva da DP (Politis, 2014). Todavia, SPECT com 99mTc-TRODAT-1 é um método caro e pouco disponível no mundo, principalmente nos países em desenvolvimento.

A USTC avalia a ecogenicidade dos gânglios da base encefálica, incluindo a SN. A hiperecogenicidade da substância negra (HSN) ocorre em 90\% dos doentes de DP idiopática e pode ser observada inclusive na fase préclínica da doença, em indivíduos que apresentam apenas sintomas não 
motores (Berg et al., 2001a). Estudo prospectivo, longitudinal e multicêntrico revelou que idosos saudáveis com HSN têm risco relativo 17 vezes maior de desenvolver DP em comparação aos idosos sem HSN (Berg et al., 2011b).

Disautonomia, hiposmia, transtorno comportamental do sono REM (TCSREM), depressão, obstipação intestinal, baixa densidade de TDA estriatal e HSN podem ser considerados biomarcadores da DP (Berg et al., 2011b). A hiposmia, caracterizada para redução da habilidade de detectar odores, é encontrada frequentemente na DP (Stenc-Bradvica et al., 2015) e pode ser detectada através de testes disponíveis e aplicáveis em ambiente ambulatorial como o teste Sniffin' Sticks de 16 ítens (SST-16).

No nosso meio, estudos a respeito da USTC (Bor-Seng-Shu et al., 2010, 2012), imagem molecular dos TDAs (Felicio AC et al., 2010; BorSeng-Shu et al., 2014a) e testes de olfação com SST-16 (Silveira-Moriyama et al., 2008) revelaram a aplicabilidade destes métodos na assistência aos doentes com DP. Entretanto, poucos estudos combinaram a USTC com teste de olfação para aumentar a acurácia no diagnóstico da DP em relação a esses métodos utilizados isoladamente, o que justifica a presente tese. 
OBJETIVOS 
Os objetivos deste estudo são:

1) Determinar a acurácia da combinação da ultrassonografia transcraniana com teste de olfação em comparação à acurácia dos referidos métodos isoladamente para o diagnóstico da Doença de Parkinson.

2) Comparar a acurácia do método combinado (ultrassonografia transcraniana e teste de olfação) com a acurácia do SPECT com TRODAT para detectar indivíduos com doença de Parkinson. 
REVISÃO DA LITERATURA 


\section{Doença de Parkinson: aspectos gerais}

Doença de Parkinson (DP) é considerada a segunda doença neurodegenerativa mais frequente da população idosa. Na população geral, a incidência anual da DP varia de 1,5 a 22 casos por 100.000 pessoas. Em indivíduos acima de 55 anos, a incidência anual varia de 410 a 529 casos por 100.000 pessoas (Wirdefeldt et al., 2011). A prevalência desta condição é de 150 a 200 casos a cada 100.000 habitantes (De Rijk et al., 2000). Na cidade de Bambuí, em Minas Gerais, a prevalência da DP em população com idade superior a 64 anos foi estimada em 3,3\% (Barbosa et al., 2006).

A DP foi primeiramente descrita em 1817 por James Parkinson no livro Essay on the Shaking Palsy. Foi denominada "paralisia agitante" em razão da tendência à inclinação anterior do tronco e cabeça, marcha em bloco, lentidão, pobreza dos movimentos e tremor de repouso. Registrou-se também neste livro os sinais e sintomas neurovegetativos e a preservação "dos sentidos e do intelecto" (Jankovic, 2008). O quadro motor predominava nas descrições iniciais dessa doença. Atualmente, a DP é considerada doença multissistêmica que acomete sistemas motores e não motores, o que pode causar prejuízo marcante na qualidade de vida. O diagnóstico da DP idiopática baseia-se na história clínica do doente, achados do exame clínico geral e neurológico, evolução clínica da doença e resultado dos exames de sangue e de neuroimagem. O diagnóstico da síndrome parkinsoniana é clínico e deve respeitar os critérios do United Kingdom Parkinson's Disease Society (Hughes et al., 1992). Recomenda-se que a bradicinesia deva estar presente como critério cardinal e que pelo menos um dos seguintes achados estejam 
presentes para confirmação da síndrome parkinsoniana: tremor de repouso, rigidez plástica ou instabilidade postural. O diagnóstico da DP idiopática requer a presença de síndrome parkinsoniana em idade apropriada, exclusão de causas secundárias de parkinsonismo e resposta terapêutica à levodopa (Hughes et al., 1992); portanto, deve-se obter dados dos exames de neuroimagem e história de exposição do paciente às substâncias tóxicas.

Os sintomas não motores da DP estão presentes desde o início da doença e apresentam aumento da prevalência com o tempo; são atualmente muito valorizados na prática clínica pois podem reforçar o diagnóstico da DP e causam prejuízo à qualidade de vida dos doentes. Constipação, transtorno de humor (por exemplo, depressão), hiposmia, ansiedade e alteração de sono (transtorno comportamental do sono REM) são considerados sintomas não motores da DP e podem ocorrer antes das anormalidades motoras clássicas. Dores, alucinações visuais, alterações urinárias e neurovegetativas, acometimento cognitivo, distúrbios do sono e dificuldade de deglutição ocorrem nos estágios tardios da doença, dificultam a socialização dos doentes e elevam os índices de institucionalização (Lauretani et al., 2014). A prevalência dos sintomas não motores da DP é de $21 \%$ no momento do diagnóstico, aumentando-se para 88\% após 7 anos. Estudo realizado no Peru, em 2013, avaliou 3000 participantes com DP e demonstrou que a maioria deles $(99,3 \%)$ apresentava ao menos um sintoma não motor. Observou-se ainda progressão considerável desses sintomas em 5 anos (Bonnet et al., 2012; Barone et al., 2009; O'sullivan et al., 2008; Shulman et al., 2001; Cosentino et al., 2013). 
Em termos fisiopatológicos, a teoria mais difundida para a DP é a hipótese de Braak (Braak et al. 2003, 2006). Segundo os autores, a formação de neuritos e corpos de Lewy (depósitos de alfa-sinucleína ou aSYN), inicia-se nas porções caudais do tronco encefálico, notadamente, no núcleo motor dorsal dos nervos glossofaríngeo e vago e também no telencéfalo, especificamente, no núcleo olfatório anterior. Nos estágios iniciais, são igualmente descritos os depósitos de alfa sinucleína (aSYN) nas fibras nervosas dos neurônios do plexo mioentérico do tubo digestivo. Estes dados justificam os sintomas não motores como hiposmia e obstipação intestinal que antecedem amiúde os sintomas motores da DP. Ulteriormente, depósitos de aSYN estenderiam-se à parte compacta da SN e, mais tardiamente, aos núcleos intralaminares talâmicos. A disseminação dessas lesões pelo neocórtex podem explicar o aparecimento de alucinações e demência nestes doentes (Braak et al., 2003, 2006).

Classicamente, a DP pode ser dividida em dois tipos clínicos: tipo tremor predominante e tipo rígido-acinética predominante. No primeiro, o tremor de repouso é o sintoma motor predominante e, no último, predomina-se a acinesia, bradicinesia e rigidez plástica (Lauretani et al., 2014). Entretanto, mais que uma simples classificação didática de duas formas clínicas, a DP apresenta um amplo espectro de apresentação clínica em virtude da grande variabilidade individual dos doentes em termos de heterogeneidade de acometimento dos circuitos neuronais (Morrison et al.,1998). A definição de subtipos clínicos da DP auxilia a predição do padrão evolutivo e prognóstico dos doentes e favorece estratégias terapêuticas personalizadas. O acometimento cognitivo, hipotensão ortostática e transtorno comportamental do 
sono REM conferem prognóstico desfavorável à progressão do declínio cognitivo e dos sintomas e sinais motores e não motores. A evolução clínica dos doentes pode ser estratificada em três grupos: grupo de progressão lenta, predominantemente motora, grupo intermediário e grupo de progressão rápida ou difusa (Fereshtehnejad et al., 2015).

\section{Ultrassonografia transcraniana na doença de Parkinson}

USTC foi inicialmente desenvolvida para avaliação vascular encefálica. Esta técnica utiliza energia de ultrassom capaz de atravessar crânio íntegro. Trata-se de um exame não invasivo, que não necessita contrastes radiológicos ou substâncias ionizantes, de baixo custo e de execução relativamente rápida. Desde meados dos anos 1990, a USTC é utilizada para visualizar o parênquima encefálico. Em 1995, Becker et al. demonstraram pela primeira vez a HSN na DP (Becker et al., 1995) (Figura 1). A HSN apresenta sensibilidade de $87,2 \%$ e especificidade de $94,9 \%$ para o diagnóstico de DP (Ressner et al., 2007). Esta técnica é empregada para o diagnóstico diferencial das anormalidades do movimento (Figura 1).

\section{Estudos transversais com USTC}

Estudos transversais evidenciaram HSN em cerca de $90 \%$ dos pacientes com DP e em $10 \%$ de indivíduos saldáveis. Doentes com rigidez e bradicinesia bilaterais apresentam área ecogênica da $\mathrm{SN}$ maior que naqueles com sintomas unilaterais (Bártová et al., 2010). A área ecogênica da SN tende 
a ser maior contralateralmente ao lado do corpo mais sintomático (Berg et al., 2001b). A USTC apresenta sensibilidade, especificidade e valor preditivo positivo elevados, respectivamente, $91,4 \%, 90 \%$ e $90,1 \%$ para o diagnóstico de DP (Budisic et al., 2009). A HSN, mensurada pela área ecogênica da região da SN, não se modifica com a progressão da doença; trata-se de um marcador da DP e não da sua progressão (Berg et al., 2005).

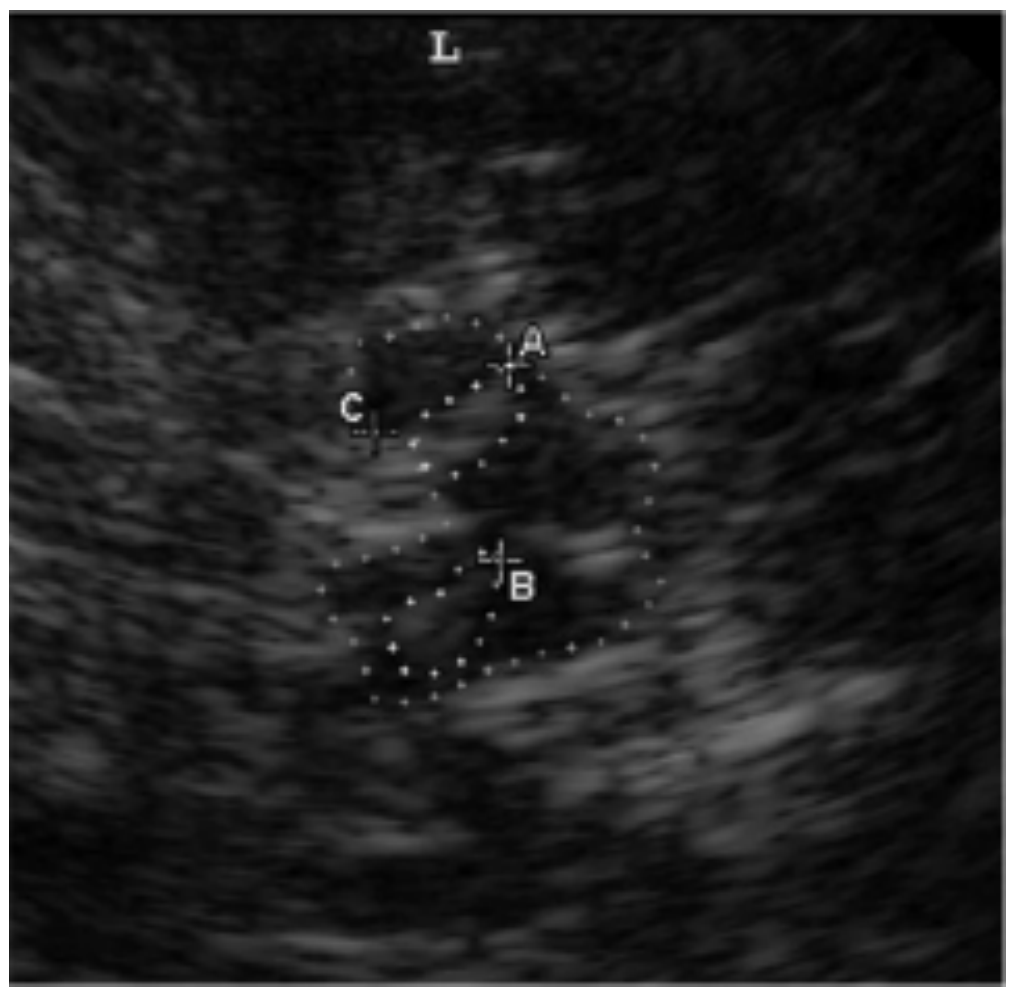

FONTE: Laboratório de Neurossonologia do HCFMUSP.

Figura 1 - Imagem da ultrassonografia do mesencéfalo. O mesencéfalo tem aspecto de uma borboleta e foi circundado (C). As regiões da substância negra igualmente circundadas (A e B)

Estudos com PET revelaram que indivíduos normais com HSN apresentam redução na captação estriatal de fluorodopa ( ${ }^{18} \mathrm{~F}$-dopa), compatível 
com vulnerabilidade funcional do sistema dopaminérgico nigroestriatal. Portanto, indivíduos saudáveis com HSN apresentam potencialmente maior vulnerabilidade a bloqueadores de receptores de dopamina e predisposição à DP (Berg et al., 2001a; Miyamoto, 2013).

\section{Estudos longitudinais com USTC}

A fase pré-clínica da DP é caracterizada pela presença de sinais e sintomas não motores como disautonomia, hiposmia, obstipação intestinal, depressão e transtorno comportamental do sono REM (TCSREM), antes do aparecimento de sintomas e sinais parkinsonianos. Publicações indicam ocorrência de HSN já na fase pré-clínica da DP (Berg, 2011a), contudo poucos estudos abordaram a HSN como fator de risco de DP.

Investigação prospectiva recente avaliou 60 doentes com parkinsonismo de causa indeterminada, submetidos inicialmente à USTC. Após 12 meses, DP foi definida em 43 doentes e SPA em 13 doentes. A USTC apresentou sensibilidade de $90,7 \%$, especificidade de $82,4 \%$ e acurácia de 88\% para predição da DP (Gaeslen et al., 2008). Pesquisa semelhante acompanhou 196 doentes com parkinsonismo de causa indefinida durante 2 anos. Demonstrou-se que apenas $52 \%$ dos doentes com HSN tiveram diagnóstico final de DP idiopática. A sensibilidade da HSN para o diagnóstico pré-clínico da DP foi de 40\% (intervalo de confiança entre 0,30 e 0,50) e a especificidade foi de $61 \%$ (intervalo de confiança variou de 0,52 a 0,70); a acurácia da USTC não foi satisfatória para predição da DP (Bouwmans et al., 2013). 
Em um estudo que incluiu 43 indivíduos com TCSREM e 149 controles saudáveis, a HSN foi encontrada em $36 \%$ dos participantes do primeiro grupo e $11 \%$ do último no início do seguimento. Indivíduos com resultados normais de USTC e ${ }^{123}$-SPECT não desenvolveram DP, enquanto que 5 indivíduos com HSN e TCSREM desenvolveram DP após 29 meses. O risco relativo para o desenvolvimento de DP em indivíduos com TCSREM e HSN foi 2,67 (Iranzo et al., 2010). Em outro estudo que incluiu 55 pacientes com TCSREM, 34,5\% deles apresentaram HSN, 58,2\% apresentaram área ecogênica da SN normal e 7,3\% não tinham janela óssea para realização do exame. Depois de 5 anos, dos 19 pacientes com HSN, 10 permaneceram saudáveis e 8 desenvolveram DP ou demência por corpos de Lewy (DCL). Dos 32 pacientes sem HSN, 21 permaneceram saudáveis e 11 deles desenvolveram DP, DCL ou atrofia de múltiplos sistemas (AMS) (Iranzo et al., 2014).

Em um trabalho que acompanhou 24 indivíduos com hiposmia submetidos à USTC, 7\% deles desenvolveram DP e 13\% anormalidades do sistema motor após 4 anos. Dos 11 pacientes que apresentavam HSN, 3 desenvolveram parkinsonismo. Nenhum dos 14 pacientes sem HSN desenvolveu alteração da função motora (Haehner et al., 2007).

Considerando depressão como manifestação pré-clínica, 57 doentes com depressão maior foram submetidos à USTC e avaliação neuropsicológica. Após 10 anos, 3 participantes $(7,5 \%)$ desenvolveram DP; verificou-se que estes já apresentavam hiposmia e HSN quando da admissão. Dezessete pacientes perderam o seguimento. Os resultados revelaram a importância da hiposmia e HSN para predição da DP (Walter et al., 2015). 
O estudo multicêntrico PRIPS (Prospective Validation of Risk Factors for the Development of Parkinsonian Syndromes) incluiu 1847 adultos saudáveis (idade $>50$ anos) e objetivou avaliar o valor da USTC na predição da DP. Após 37 meses, os adultos saudáveis com HSN apresentaram risco 17 vezes maior de desenvolver DP, comparados ao grupo sem HSN. Concluiu-se que, em adultos saudáveis, a HSN é fator de risco para o desenvolvimento de DP. Outros biomarcadores de fase pré-clínica da DP como depressão, hiposmia e TCSREM apresentaram risco relativo menor em relação à HSN (Berg et al., 2011b). A mesma coorte de participantes foi reavaliada após 5 anos; nessa fase, dos 1271 indivíduos restantes, aqueles com HSN apresentaram risco 20 vezes maior de desenvolver DP em relação ao grupo sem HSN. A área ecogênica da SN não aumentou neste período (Berg et al., 2013; Lerche et al., 2014).

\section{Imagem molecular na doença de Parkinson}

Nas últimas décadas, a avaliação da DP objetivou o enfoque estrutural, funcional e molecular do encéfalo. A caracterização multimodal das alterações neurobiológicas na DP demonstra a heterogeneidade clínica e fisiopatológica desta condição e contribui para identificar novas estratégias de terapias medicamentosa e cirúrgica. A Ressonância nuclear magnética (RM) encefálica e a imagem molecular dos TDAs auxiliam na determinação precoce do diagnóstico da DP e no diagnóstico diferencial das síndromes neurodegenerativas. A neuroimagem molecular é obtida através das técnicas de PET e SPECT (Politis, 2014). 
A função dopaminérgica pré-sináptica no striatum pode ser avaliada através de radiotraçadores com afinidade a moléculas relacionadas a turnover de dopamina nos neurônios da SN tais como a dopa-descarboxilase (que realiza conversão de L-dopa em dopamina), os TDA (responsáveis pela captação da dopamina na terminação pré-sináptica) e os transportadores vesiculares de monoaminas (que armazenam e liberam dopamina sintetizada). A princípio, déficits dopaminérgicos pré-sinápticos estriatais primários predominantemente unilaterais são indicativos de DP idiopática e déficits póssinápticos primários sugerem SPA (Seibyl, 2003).

Transportadores de dopamina (TAD) são moléculas que captam a dopamina nas terminações pré-sinápticas (Piccini et al., 2003) dos dendritos e axônios dos neurônios dopaminérgicos (Boulton; Eisenhofer, 1998). A quantidade de TDA estriatal correlaciona-se com o número de terminações dopaminérgicas pré-sinápticas. Os radiotraçadores com afinidade a TDA são: TRODAT-1, FP-CIT e $\beta$-CIT, para exames de SPECT, e $\beta$-CFT e FE-CIT, para exames de PET (Pirker et al., 2000; Tissingh et al., 1997).

Níveis reduzidos de TDA (Figura 2) são observados em pacientes com DP, demência associada à DP, paralisia supranuclear progressiva (PSP) e MSA (Politis, 2014). SPECT para avaliação dos TDA tem alta sensibilidade (87-98\%) e especificidade (80-100\%) para diferenciar pacientes com síndromes parkinsonianas daqueles com TE ou controles saudáveis (Figura 2). SPECT com radiotraçadores para TDA pode diferenciar doentes com DP de indivíduos saudáveis com sensibilidade de 90\% (Pirker et al., 2000; Tissingh et al., 1997). Contudo, a quantificação dos TDA encefálicos através de SPECT não é adequada para diferenciar DP de outras SPA visto que o acometimento da via 
dopaminérgica nigroestriatal ocorre tanto na DP como nos parkinsonismos atípicos (Varrone et al., 2001).
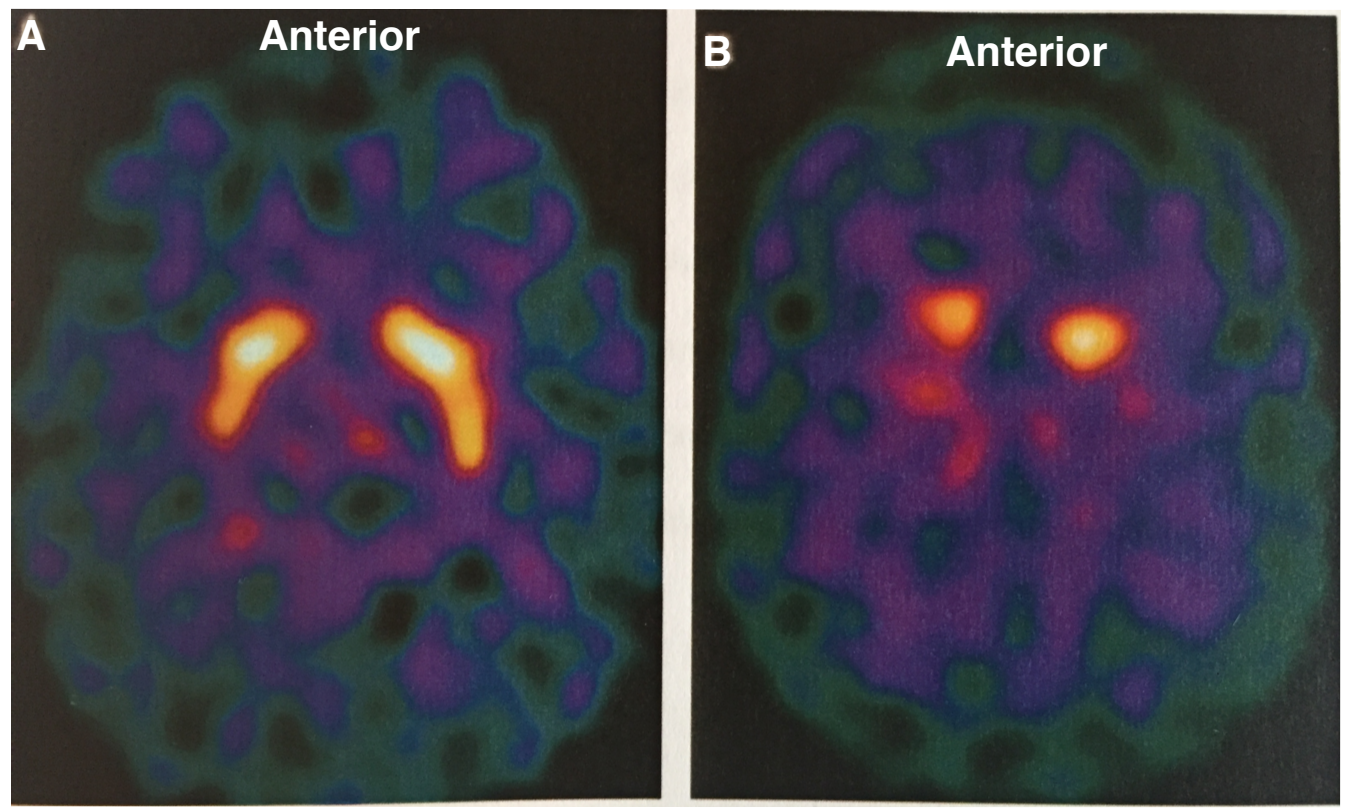

FONTE: Bor-Seng-Shu, 2012.

Figura 2 - SPECT encefálico com 9mTc-TRODAT-1 de um indivíduo saudável (A) e de um indivíduo com doença de Parkinson (B). Nota-se redução significativa de captação estriatal do radiotraçador no indivíduo com doença de Parkinson

\section{Olfação na doença de Parkinson}

Os primeiros relatos de perda do sentido do olfato na DP foram publicados em 1975 por Ansari e Johnson (Ansari; Johnson, 1975). Atualmente, hiposmia é considerado sintoma não motor da DP, de alta prevalência, acometendo até $90 \%$ desses doentes. A anormalidade do olfato pode preceder os sintomas motores da DP e é considerada marcador biológico de sinucleinopatia. A avaliação do olfato é útil e viável na prática médica diária, ao contrário dos métodos que avaliam degeneração da via nigroestriatal (Ross et 
al., 2008; Xiao et al., 2014). No Brasil, a validação e adaptação dos testes de olfato para DP foram feitas pelo Grupo de Distúrbios do Movimento do hospital das clínicas da faculdade de medicina da universidade de São Paulo (HCFMUSP) em 2008. Na ocasião, aplicou-se o teste de identificação de odores de 40 itens da Universidade da Pensilvânia e o teste Sniffin' Stick (Figura 3) em indivíduos saudáveis e, igualmente, nos pacientes com DP. O primeiro teste apresentou sensibilidade de $82,1 \%$ e especificidade de $83,5 \%$ para a detecção da DP e o último teste identificou DP com sensibilidade de $81,1 \%$ e especificidade de $89 \%$ (Silveira-Moriyama et al., 2008).

Para o diagnóstico da DP, a especificidade dos métodos que avaliam a função olfativa é maior que a sensibilidade porque hiposmia pode ocorrer também em outras condições clínicas como a doença de Alzheimer. Por outro lado, doentes com PSP, AMS, degeneração córticobasal (DCB) e TE apresentam função olfatória normal (Wenning et al., 1995). Para verificar o papel da hiposmia na predição da DP, 361 indivíduos assintomáticos foram acompanhados durante 2 anos. Avaliação clínica inicial revelou hiposmia em 40 participantes. Após 2 anos, $10 \%$ dos participantes com hiposmia desenvolveram DP. Todos os pacientes que desenvolveram DP também apresentavam, desde o início do projeto, redução da quantidade de TDA estriatal (Ponsen et al., 2004). O desempenho em testes de olfação também apresentou correlação com a captação do TAD no putamen e estriado de pacientes com DP avaliados ateavés de exames de SPECT com 99mTcTRODAT-1 (Siderowf et al., 2005). 


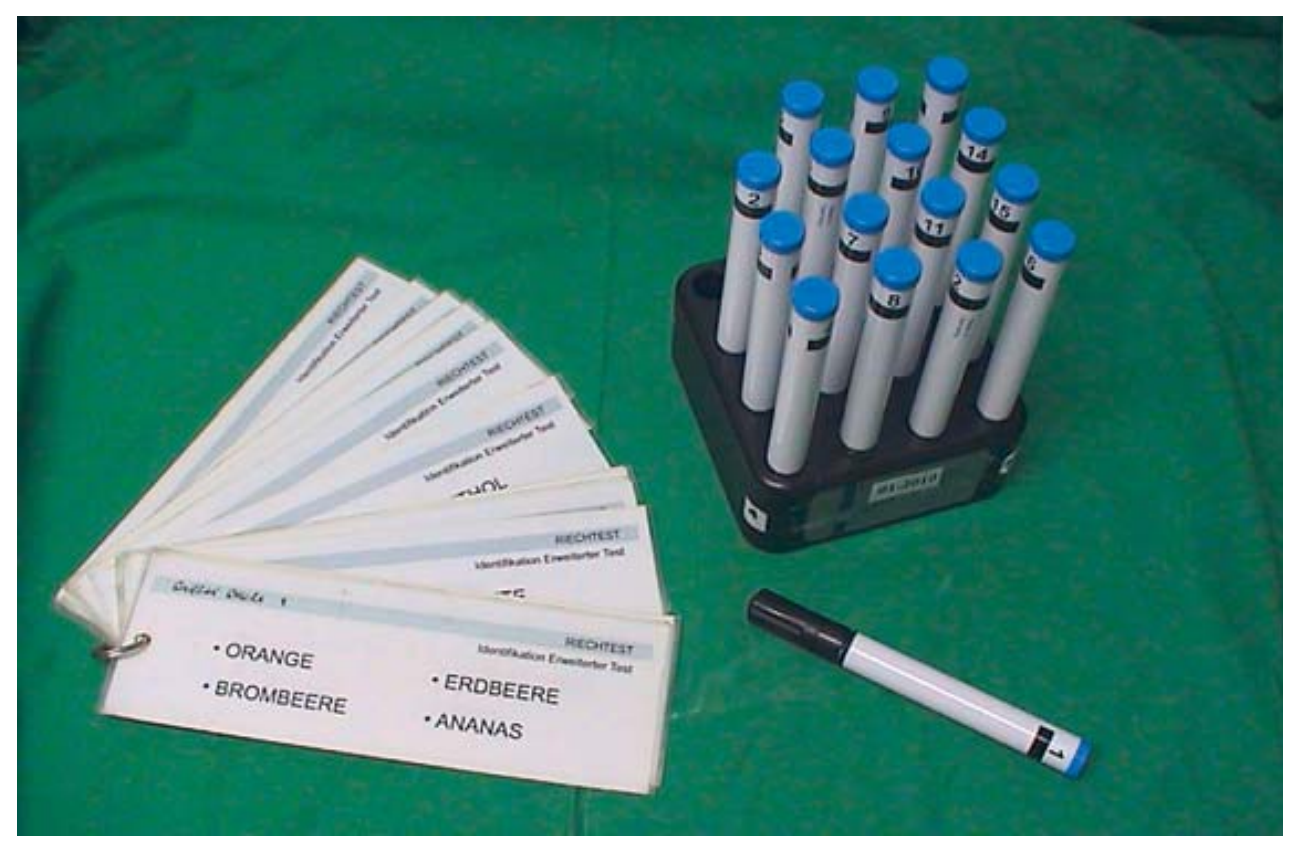

FONTE: Hüttenbrink KB, et al., 2013.

Figura 3 - Teste de Sniffin'Sticks com 16 itens. Canetas dispensadoras de odores são expostas ao paciente e cada odor deve ser identificado em uma lista de quatro itens

Um outro estudo avaliou pacientes com DP através de exames de imagem molecular, USTC e testes para odores. Houve correlação entre o desempenho no teste de olfação e o estágio clínico da DP aferido através da escala UPDRS (Unified Parkinson's disease rating scale). Da mesma foram, houve correlação entre o desempenho no teste de olfação e a área de ecogenicidade da SN e a capacidade de armazenamento de dopamina aferida através de PET com ${ }^{18}$ F-DOPA (Scherfler et al., 2013).

\section{Considerações finais}

O diagnóstico diferencial entre DP e outras SPA é difícil, principalmente nas fases iniciais quando se manifesta apenas a síndrome 
parkinsoniana, comum a todas as condições. A definição diagnóstica precoce possibilita predição do prognóstico, orientação ao paciente e escolha apropriada do tratamento. O diagnóstico precoce, sobretudo em fase préclínica das doenças, permitiria melhor eficácia das terapias neuroprotetoras, antes que houvesse degeneração significativa do tecido neural. $O$ reconhecimento dos mecanismos fisiopatogênicos das doenças permitiria intervenção específica e, consequentemente, modificação do processo de neurodegeneração. Por estas razões, os métodos diagnósticos devem ter acurácia, sensibilidade e especificidade reconhecidas pelo clínico. O mesmo poderá decidir qual melhor método a ser indicado para cada situação clínica. A indisponibilidade ou o alto custo de um método diagnóstico deve incentivar estratégias diagnósticas que combinem métodos mais acessíveis e baratos para diagnóstico acurado da DP, ou para a diferenciação entre DP e SPA. O diagnóstico diferencial não pode preterir a história e o exame neurológico cuidadosos. Aliado a isso, a combinação de métodos diagnósticos pode revelar a importância dos marcadores biológicos apresentados nesta tese devido às suas disponibilidades e custos nas diversas regiões do mundo. 
MÉTODOS 


\section{Aspectos Éticos}

A pesquisa obedeceu às normas estabelecidas pela Resolução $n^{\circ}$ 466/2012 do Conselho Nacional de Saúde e foi aprovada pelo Comitê de Ética em pesquisa do Hospital das Clínicas da Faculdade de Medicina da Universidade de São Paulo (HCFMUSP). Todos os participantes assinaram termo de consentimento livre e esclarecido.

\section{Desenho da pesquisa e local de realização}

Trata-se de uma pesquisa transversal, observacional, descritiva e analítica em que os participantes foram avaliados em 3 locais: 1) Setor de Distúrbios do Movimento da Universidade Federal de São Paulo (UNIFESP), 2) Setor de Medicina Nuclear do Instituto de Ensino e Pesquisa do Hospital Israelita Albert Einstein (HIAE) e 3) Laboratório de Neurossonologia do HCFMUSP,

\section{Amostra da pesquisa e critérios de inclusão e exclusão}

A população avaliada foi recrutada no ambulatório de distúrbios do movimento da UNIFESP e organizada em dois grupos: o grupo caso, constituído de doentes com DP, e o grupo controle, formado por indivíduos saudáveis. Foram incluídos pacientes com diagnóstico de DP idiopática baseado nos critérios do United Kingdom Parkinson's Disease Society (Gibbs, Lees, 1988; Hughes et al., 1992). O grupo controle consistiu de indivíduos 
saudáveis, funcionários da UNIFESP, que foram igualmente entrevistados e avaliados pela equipe do Setor de Distúrbios do Movimento da mesma universidade. Ambos os grupos foram pareados em função da idade e gênero. Excluiu-se os participantes que apresentavam história familiar de parkinsonismo, exposição às drogas neurolépticas, suspeita de SPA, tabagismo, infecção ativa de via respiratória superior, rinossinusite crônica, transtornos psiquiátricos, traumatismo cranioencefálico com perda de consciência e desempenho insatisfatório no mini-exame do estado mental (MMEE), este último segundo notas de corte validadas para a população brasileira (Brucki et al., 2003).

\section{Coleta de dados}

As variáveis clínico-demográficas coletadas foram: idade, gênero, tempo de escolaridade, idade ao início dos sintomas e duração da doença. Participantes com DP foram examinados na fase off através da escala Hoehn e Yahr modificada (HY), parte III do UPDRS e escala de Schwab e England (SE). Para ambos os grupos, utilizou-se MMEE normalizado para língua portuguesa falada no Brasil (Brucki et al., 2003) e o inventário de depressão de Beck na versão portuguesa validada para o Brasil (Gorestein; Andrade, 1996). A seguir, os participantes foram encaminhados para os seguintes procedimentos: SPECT com TRODAT, teste de olfação com técnica SST-16 e, por fim, USTC. SST-16 e SPECT foram realizados nos anos de 2008 e 2009 e USTC no início de 2010; o intervalo entre SST-16/SPECT e USTC variou de 6 a 23 meses. Os 
pesquisadores que realizaram os exames de imagem molecular e USTC não tiveram acesso aos dados clínicos dos participantes.

\section{Ultrassonografia transcraniana}

A USTC foi realizada por um examinador experiente (Bor-SengShu), que não teve acesso aos dados clínicos e dos testes complementares dos participantes. Utilizou-se um equipamento de ultrassonografia acoplado a transdutores setoriais (phased-array) de 5-1 MHz (modelo MicroMaxx® da empresa Sonosite, USA). Os exames foram realizados conforme diretrizes da Sociedade Europeia de Neurossonologia e Hemodinâmica Cerebral (Walter et al., 2007a). Em casos de janela acústica inadequada, mensurou-se a área ecogênica da SN de ambos os lados através da janela disponível.

\section{SPECT com TRODAT}

As imagens de SPECT foram obtidas após 4 horas da injeção de $814 \mathrm{MBq}( \pm 74)$ de 99mTc-TRODAT-1, produzido pelo Instituto de pesquisa em Energia Nuclear de Taiwan. As drogas antiparkinsonianas foram suspensas cerca de 12 horas dos exames de SPECT. Utilizou-se câmaras de duas cabeças, acopladas a colimadores de furos divergentes (Infinia ${ }^{\mathrm{TM}}$ Hawkeye ${ }^{\circledR}$ SPECT/CT, GE Healthcare). A solução de 99mTc-TRODAT-1 foi preparada, rotulada e reavaliada, em termos de grau de pureza do radiotraçador através do método de extração de solvente. As imagens foram reconstruídas e reordenadas com referência à linha formada entre as comissuras anterior e 
posterior, na estação de trabalho Xeleris (GE Medical Systems). Para correção da atenuação, utilizou-se o método de Chang de primeira ordem (Chang, 1978). Dois avaliadores independentes, igualmente sem conhecimento dos dados clínicos dos participantes, delimitaram regiões de interesse nos núcleos estriados, núcleos caudados, putamens e lobos occipitais. A densidade de ligação do TRODAT ao TDA foi obtida nas regiões de interesse através da equação (ligação específica - ligação inespecífica)/ligação inespecífica.

\section{Teste de olfação}

A avaliação da olfação baseou-se no método SST-16 (Burghart Medizintchnik, Alemanha), em sua versão validada para a língua portuguesa brasileira (Santin et al., 2010; Silveira-Moriyama et al., 2008). Esse exame expõe aos participantes 16 odores distintos: de laranja, couro, canela, hortelã, banana, limão, alho, café, maçã, cravo, abacaxi, rosa, peixes, adocicado, óleo de madeira (ou terebentina) e ervas. As canetas que exalam odor têm $14 \mathrm{~cm}$ de comprimento, funcionam, em média, um ano e devem ser utilizadas em sala ventilada e silenciosa. Para cada odor, o examinador aproxima a ponta da caneta a cerca de $2 \mathrm{~cm}$ das narinas dos participantes, os quais devem identificar o odor em uma lista de quatro opções. Quando o odor não é identificado, o participante é orientado a escolher o mais provável dentre as opções dadas. O SST-16 afere a identificação, o limiar e a discriminação dos odores. Definiu-se a quantidade de acertos na identificação dos odores como a variável que mensura o grau de acometimento olfatório. 


\section{Análise Estatística}

Aplicou-se o teste de Shapiro-Wilk para verificar a distribuição normal dos dados. As variáveis paramétricas foram descritas em termos de média e desvio padrão (DP), enquanto que as variáveis não paramétricas foram descritas em termos de mediana e quartis ou percentis 25 e 75 . O teste de Qui-quadrado e os seus demais testes complementares foram utilizados para correlacionar variáveis categóricas. As variáveis contínuas com distribuição normal foram avaliadas pelo teste $t$ de Student. Para as variáveis contínuas não paramétricas, usou-se o teste de Mann-Whitney U. O coeficiente de Spearman`s rho foi utilizado para correlação bivariada entre variáveis não paramétricas. Para avaliar a concordância entre os métodos diagnósticos, utilizou-se o teste Kappa considerando SPECT com 99mTc-TRODAT como referência. Curvas ROC foram utilizadas para identificação dos pontos de corte para detecção de DP na USTC, SPECT e SST-16. A acurácia dos métodos USTC, SPECT e SST-16 foi calculada através da área sob a curva ROC. Ulteriormente, calculou-se a acurácia combinada dos métodos USTC e SST-16 (alteração em um ou outro método). O erro alfa foi estabelecido em 0,05 e o intervalo de confiança em $95 \%$. A análise estatística foi realizada através do programa "SPSS 20.0 for Windows". Para análise estatística dos dados da USTC, considerou-se o maior valor das áreas ecogênicas da SN de ambos os lados. Para SPECT com TRODAT, optou-se pelo menor valor dos potenciais de ligação estriatal de ambos os lados. 
RESULTADOS 


\section{Características da amostra}

A amostra consistiu de 20 doentes com DP (13 homens e 7 mulheres) e 9 indivíduos saudáveis (6 homens e 3 mulheres). A mediana da idade foi de 51 (46-54) no grupo DP e 62,5 (54-67,5) no grupo controle. Comparação destes grupos não revelou diferença significativa em termos de idade e gênero. Os dados demográficos foram descritos na Tabela 01.

Tabela 1 - Distribuição dos dados demográficos e clínicos entre os indivíduos do grupo controle e do grupo com doença de Parkinson (caso)

\begin{tabular}{|c|c|c|c|c|}
\hline Dados & $\mathrm{N}$ & Média \pm DP & $\begin{array}{c}\text { Mediana }(25- \\
75)\end{array}$ & $\begin{array}{l}\text { Valor } \\
\text { "p" }\end{array}$ \\
\hline $\begin{array}{r}\text { Gênero (M/F) } \begin{array}{r}\text { Controle } \\
\text { Caso }\end{array}\end{array}$ & $\begin{array}{c}6 / 3 \\
13 / 7\end{array}$ & & - & $0,930 \mathrm{a}$ \\
\hline $\begin{aligned} \text { Idade(anos) Controle } & <65 \\
& \geq 65 \\
\text { Caso } & <65 \\
& \geq 65\end{aligned}$ & $\begin{array}{c}9 \\
0 \\
13 \\
7\end{array}$ & & - & $0,066 a$ \\
\hline $\begin{array}{l}\text { Escolaridade (anos) } \\
\text { Controle } \\
\text { Caso }\end{array}$ & & $\begin{array}{c}13,00 \\
5,5\end{array}$ & $\begin{array}{c}13(10-15,5) \\
5,5(4-12)\end{array}$ & $0,237 \mathrm{~b}$ \\
\hline $\begin{array}{l}\text { Idade início dos } \\
\text { sintomas(anos) }\end{array}$ & & $59,25 \pm 13,41$ & $56,5(49-66,7)$ & - \\
\hline $\begin{array}{l}\text { Duração da doença } \\
\text { (anos) }\end{array}$ & & $4,70 \pm 3,04$ & $5,0(2,2-5,7)$ & 一 \\
\hline UPDRS III & & $28,60 \pm 14,57$ & $\begin{array}{c}29,0 \\
(17,5-35,5)\end{array}$ & - \\
\hline $\begin{array}{r}\text { Controle } \\
\text { Caso }\end{array}$ & & $\begin{array}{c}5,77 \pm 3,27 \\
10,30 \pm 3,82\end{array}$ & - & $0,071 \mathrm{c}$ \\
\hline $\begin{array}{r}\text { Controle } \\
\text { Caso }\end{array}$ & & $\begin{array}{l}27,66 \pm 1,80 \\
26,00 \pm 3,59\end{array}$ & - & $0,202 \mathrm{c}$ \\
\hline
\end{tabular}

FONTE: Laboratório de Neurossonologia do HCFMUSP.NOTA: DP: desviopadrão, F: Feminino, M: Masculino, MMEE: Miniexame do estado mental, Quiquadrado a, Teste de Mann-Whitneu $\mathrm{U}^{\mathrm{b}}$, Teste T-student ${ }^{\mathrm{c}}$. 
O desempenho na escala UPDRS III apresentou mediana de 29 $(17,5-35,5)$ e, na escala HY, mediana de $2,2(2,0-2,5)$. Um paciente encontravase no estágio 1, 9 pacientes no estágio 2, 9 pacientes no estágio 3 e 1 paciente no estágio 4. No tocante à escala SE, $58,6 \%$ dos pacientes apresentavam-se com escores entre 80 e 100 pontos.

\section{Área ecogênica em região da SN}

A área ecogênica da $\mathrm{SN}$ foi significativamente maior no grupo DP do que no grupo controle $(p=0,013)$ (Tabela 2). Análise da curva ROC (Gráfico 1) revelou que a área ecogênica da $\mathrm{SN}$ de $0,22 \mathrm{~cm}^{2}$ associou-se à sensibilidade de $100 \%$ e especificidade de $77,8 \%$ para o diagnóstico de DP (Tabela 3). Todos os participantes com DP apresentaram área ecogênica da SN maior que o referido valor de corte, enquanto que, do grupo controle, somente 2 participantes. A acurácia da USTC para detecção de DP foi de 79,2\% (Tabela 4). Dois participantes apresentaram dificuldade para visualização mesencefálica, um deles com janela inadequada à direita e outro à esquerda.

\section{Captação estriatal de ${ }^{99 m}$ Tc-TRODAT}

A captação estriatal de 99mTc-TRODAT foi significativamente menor no grupo DP que no grupo controle $(p=0,001)$ (Tabela 2). Através da curva ROC (Gráfico 3), estabeleceu-se o valor de corte de 0,90 para o diagnóstico de DP (sensibilidade de 100\% e especificidade de $88,9 \%$ ). Todos 
os pacientes com DP apresentaram baixa captação estriatal de ${ }^{99 m}$ Tc-TRODAT (valor de potencial de ligação menor que o valor de corte), enquanto que este achado foi observado apenas em um participante saudável $(p=0,001)$. Para o diagnóstico de DP, as acurácias da captação de 99mTc-TRODAT no striatum, núcleo caudado e putamen foram, respectivamente, 99\%, 97,5\% e 94,4\% (Tabela 4).

Tabela 2 - Descrição dos valores obtidos na avaliação por ultrassonografia transcraniana, SPECT com TRODAT e teste de percepção olfatória no grupo de participantes controles e com doença de Parkinson

\begin{tabular}{lccccccc}
\hline Dados & \multicolumn{3}{c}{ Grupo controle } & \multicolumn{2}{c}{ Doença de Parkinson } & $\begin{array}{c}\text { Valor } \\
\text { "p" ** }\end{array}$ \\
& 25 & Mediana & 75 & 25 & Mediana & 75 & \\
\hline $\begin{array}{c}\text { Ecogenicidade da } \\
\text { SN (area/cm2) }\end{array}$ & 0,14 & 0,17 & 0,43 & 0,40 & 0,51 & 0,65 & 0,013 \\
& & & & & & & \\
$\quad \begin{array}{c}\text { Captação } \\
\text { estriatal de } \\
\text { TRODAT }\end{array}$ & 1,03 & 1,18 & 1,41 & 0,47 & 0,58 & 0,67 & 0,001 \\
$\begin{array}{c}\text { Desempenho no } \\
\text { teste de olfação }\end{array}$ & 11,00 & 12,00 & 13,00 & 5,25 & 9,00 & 10,75 & 0,001 \\
\hline
\end{tabular}

FONTE: Laboratório de Neurossonologia do HCFMUSP.

NOTA: SN: Dados apresentados em mediana e quartis. Substância Negra, TRODAT: SPECT com avaliação do transportador de dopamina com ${ }^{99 m T c, ~ * *}$ Teste Mann-Whitney U.

\section{Percepção olfatória}

O desempenho da percepção olfatória ao teste SST-16 foi pior no grupo DP que no grupo controle $(p=0,001)$ (Tabela 2). Considerando a curva 
ROC (Gráfico 2), verificou-se que o valor de corte de 10 associou-se com sensibilidade de $65 \%$ e especificidade de $100 \%$ para detecção de DP. Hiposmia foi observada em 13 (65\%) doentes com DP e em nenhum dos participantes saudáveis. A acurácia do teste SST-16 para o diagnóstico de DP foi de $85,8 \%$ $(p=0,002)($ Tabela 4).

Tabela 3 - Pontos de corte obtidos para a ultrassonografia transcraniana, SPECT com TRODAT, e teste de percepção olfatória no grupo de pacientes controles e com doença de Parkinson (caso)

\begin{tabular}{|c|c|c|c|c|c|}
\hline \multirow[t]{2}{*}{ Dados } & \multicolumn{5}{|c|}{ Grupo } \\
\hline & $\begin{array}{l}\text { Pontos de } \\
\text { corte }\end{array}$ & & Controle & Caso & $\begin{array}{l}\text { Valor } \\
\text { "p"** }\end{array}$ \\
\hline \multirow[t]{2}{*}{$\begin{array}{l}\text { Ecogenicidade da } \\
\text { SN (area/cm2) }\end{array}$} & $\geq 0,22$ & $\begin{array}{l}\mathrm{N} \\
\%\end{array}$ & $\begin{array}{c}2 \\
22,2 \%\end{array}$ & $\begin{array}{c}20 \\
100,0 \%\end{array}$ & 0,001 \\
\hline & $<0,22$ & $\begin{array}{l}\mathrm{N} \\
\%\end{array}$ & $\begin{array}{c}7 \\
77,8 \%\end{array}$ & $\begin{array}{c}0 \\
0 \%\end{array}$ & \\
\hline \multirow[t]{2}{*}{$\begin{array}{c}\text { Captação estriatal } \\
\text { de TRODAT }\end{array}$} & $\geq 0,90$ & $\begin{array}{l}N \\
\%\end{array}$ & $\begin{array}{c}8 \\
88,9 \%\end{array}$ & $\begin{array}{c}0 \\
0 \%\end{array}$ & 0,001 \\
\hline & $<0,90$ & $\begin{array}{l}\mathrm{N} \\
\%\end{array}$ & $\begin{array}{c}1 \\
11,1 \%\end{array}$ & $\begin{array}{c}20 \\
100 \%\end{array}$ & \\
\hline \multirow[t]{2}{*}{$\begin{array}{l}\text { Desempenho no } \\
\text { teste de olfação }\end{array}$} & $\geq 10$ & $\begin{array}{l}\mathrm{N} \\
\%\end{array}$ & $\begin{array}{c}9 \\
100,0 \%\end{array}$ & $\begin{array}{c}7 \\
35,0 \%\end{array}$ & 0,001 \\
\hline & $<10$ & $\begin{array}{l}\mathrm{N} \\
\%\end{array}$ & $\begin{array}{c}0 \\
0 \%\end{array}$ & $\begin{array}{c}13 \\
65,0 \%\end{array}$ & \\
\hline
\end{tabular}

FONTE: Laboratório de Neurossonologia do HCFMUSP.

NOTA: Dados expressos em frequência absoluta (n) e relativa (\%). SN: Substância Negra, TRODAT: SPECT com avaliação do transportador de dopamina com ${ }^{99 m}$ Tecnécio, ** Teste do Qui-quadrado. 
Gráfico 1 - Curva ROC considerando ultrassonografia transcraniana como método diagnóstico para Doença de Parkinson

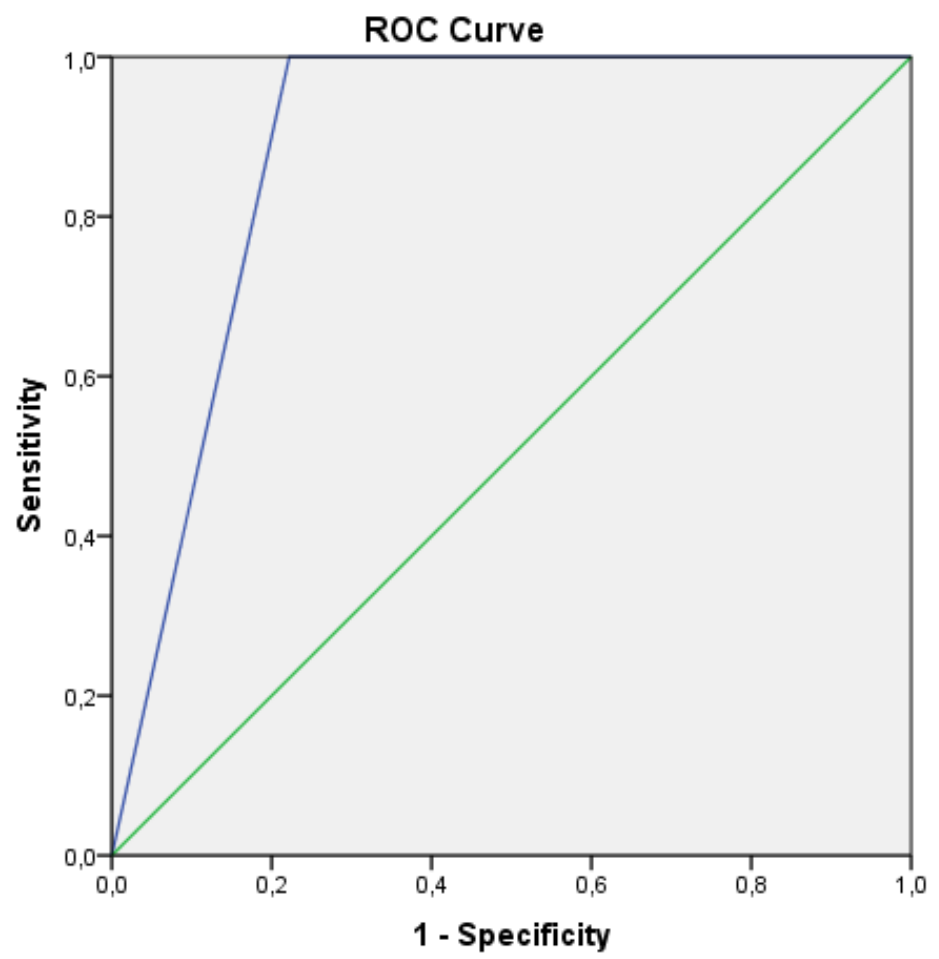

Gráfico 2 - Curva ROC considerando teste de olfação (Sniffin' Sticks com 16 itens) como método diagnóstico para Doença de Parkinson

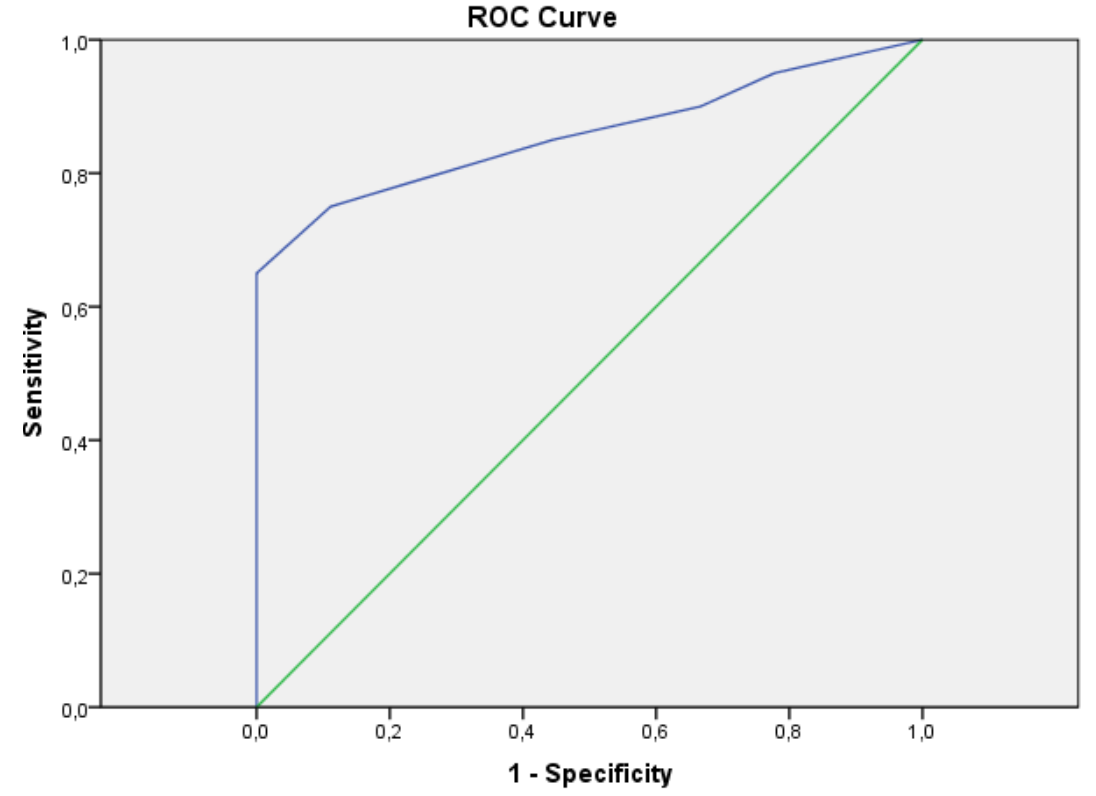


Gráfico 3 - Curva ROC considerando captação estriatal de TRODAT como método diagnóstico para Doença de Parkinson

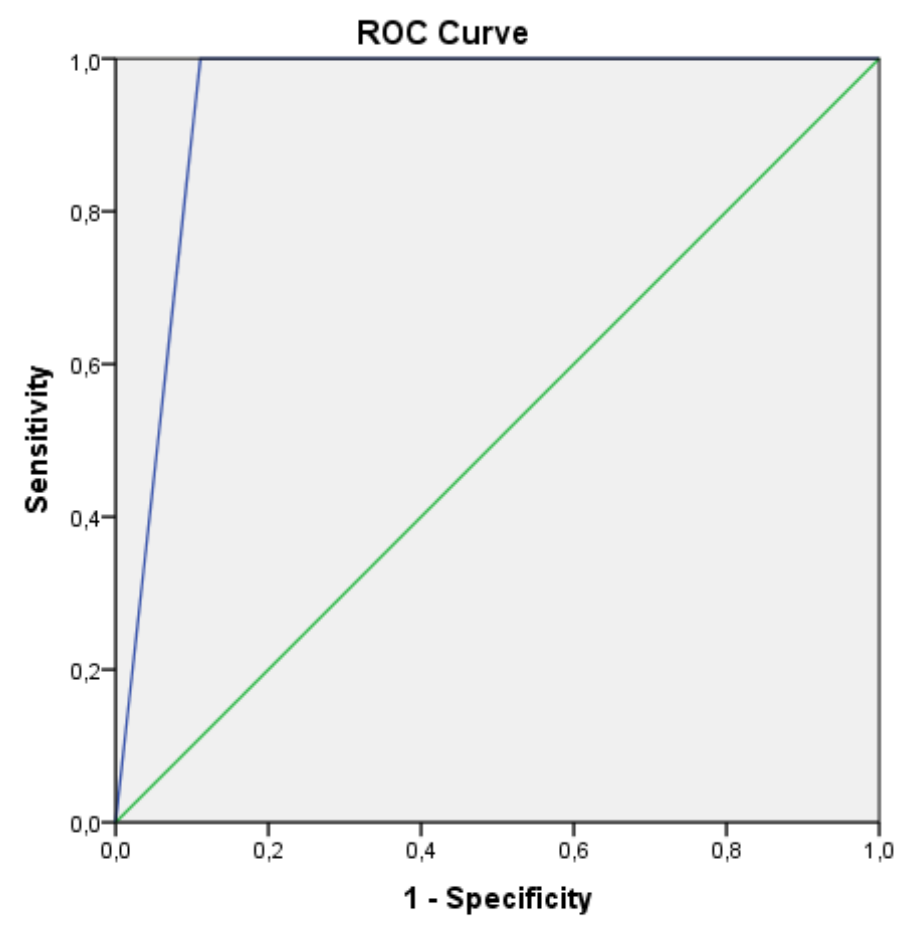

Gráfico 4 - Curva ROC considerando a combinação da ultrassonografia transcraniana e teste de olfação como método diagnóstico para Doença de Parkinson

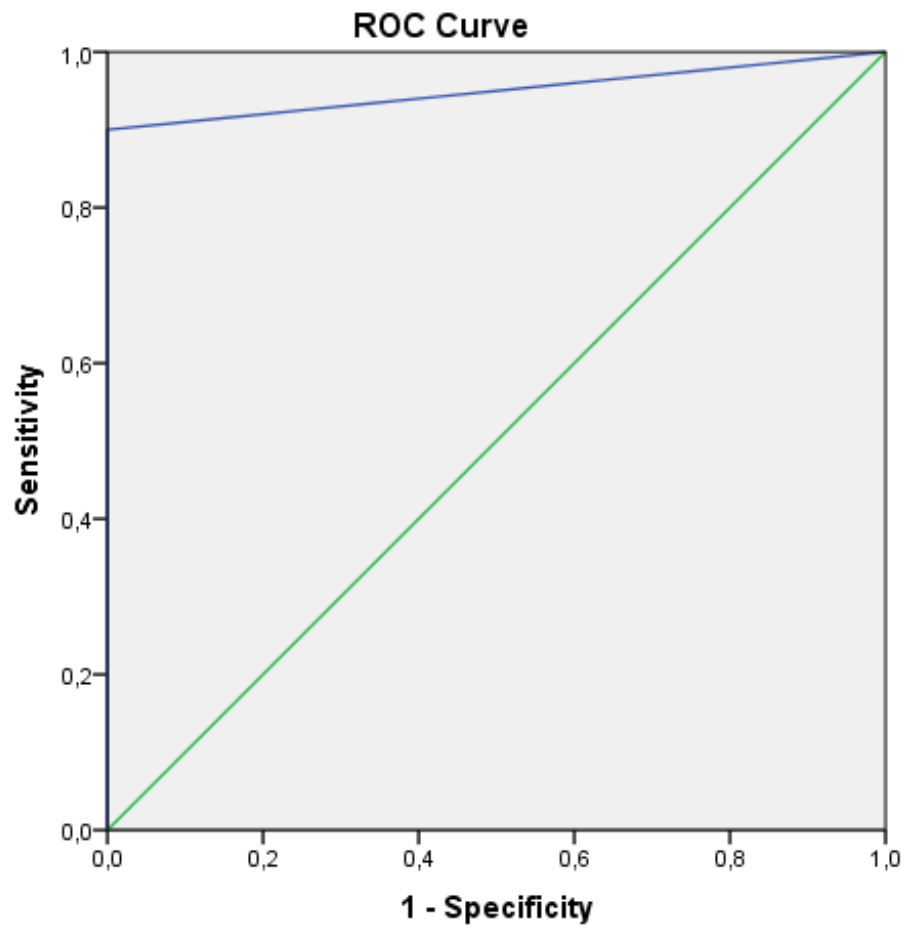


Tabela 4 - Acurácia isolada da ultrassonografia transcraniana, SPECT com TRODAT e teste de olfação isoladamente e acurácia da combinação entre ultrassonografia transcraniana e teste de olfação para o diagnóstico de doença de Parkinson

\begin{tabular}{lcccc}
\hline Dados & Área & IC $<95 \%$ & IC>95\% & Valor "p" ** \\
\hline $\begin{array}{c}\text { Ecogenicidade da SN } \\
\text { (area/cm2) }\end{array}$ & 0,792 & 0,536 & 1,001 & 0,013 \\
$\begin{array}{c}\text { Desempenho no teste de } \\
\text { olfação }\end{array}$ & 0,858 & 0,725 & 0,992 & 0,002 \\
$\begin{array}{c}\text { Captação estriatal de } \\
\text { TRODAT }\end{array}$ & 0,998 & 0,982 & 1,001 & 0,001 \\
$\begin{array}{c}\text { Desempenho no teste de } \\
\text { olfação combinado com } \\
\text { USTC }\end{array}$ & 0,95 & 0,869 & 1,000 & 0,001 \\
\hline
\end{tabular}

FONTE: Laboratório de Neurossonologia do HCFMUSP.

NOTA: Dados expressos em termos de área sob a curva ROC com seus respectivos intervalos de confiança. SN: Substância Negra, TRODAT: SPECT com avaliação do transportador de dopamina com 99mTc, USTC: ultrassonografia transcraniana, ** Teste de significância assintótica.

Comparação entre os métodos para o diagnóstico de Doença de Parkinson

Não se observou correlação entre os potenciais de ligação do radiotraçador no striatum e a área de ecogenicidade da $\mathrm{SN}(\mathrm{r}=-0,269$, $p=0,159)$. Houve correlação positiva entre os potenciais de ligação do ${ }^{99 m T c-}$ TRODAT no striatum e o desempenho da função olfatória ao SST-16 ( $r=0,423$, $p=0,022)$. Notou-se correlação negativa entre o desempenho da percepção olfatória e a área ecogênica da $S N(r=-0,423, p=0,019$; teste Spearman's rho). 
A concordância entre os resultados do SPECT com 99mTcTRODAT e os resultados da USTC foi excelente, com índice Kappa de 0,92 (erro padrão=0,77; $p<0,001)$. Concordância satisfatória foi observada entre os resultados do teste SST-16 e o método de referência, com índice Kappa de 0,518 (erro padrão $=0,132 ; p<0,001$ ).

SPECT com 99mTc-TRODAT foi o método que apresentou maior acurácia para o diagnóstico de DP e o SST-16 apresentou acurácia discretamente maior que a da USTC. A curva ROC, resultante da combinação de SST-16 com USTC (Gráfico 4), mostrou que a acurácia da combinação destes métodos se aproximou daquela observada no exame isolado de SPECT com 99mTc-TRODAT (Tabela 4). Quando os resultados dos exames SST-16 e USTC foram discordantes, SPECT com ${ }^{99 m T c-T R O D A T ~ a u x i l i o u ~ n a ~ c o n f i r m a c ̧ a ̃ o ~}$ do diagnóstico de DP. Por outro lado, resultados concordantes de SST-16 e USTC confirmaram os resultados de SPECT com 99mTc-TRODAT. 
DISCUSSÃO 
O presente estudo demonstrou aumento da área ecogênica da SN, redução da densidade estriatal dos TDA e diminuição da percepção olfatória nos doentes com DP, comparados a participantes saudáveis, e determinou os valores de corte destas variáveis para a identificação da DP. SPECT com 99mTc-TRODAT foi o método de maior acurácia para o diagnóstico da DP. SST-16 apresentou acurácia maior que a da USTC. A acurácia da combinação SST-16 e USTC aproximou-se daquela observada no SPECT com 99mTc-TRODAT. Embora a HSN, o deficit dopaminérgico da via nigroestriatal e a hiposmia tenham sido previamente demonstrados na DP (Bor-Seng-Shu et al., 2012, 2014a, 2014b; Budisic et al., 2009; Shih et al., 2007; Walter et al., 2003, 2007b; Wang et al., 2005; Wu et al., 2011) e que a combinação das avaliações olfatória e ultrassonográfica eleva a sensibilidade e especificidade do diagnóstico da DP (Izawa et al., 2012; Lopez-Hernández et al., 2015; Tunc et al., 2015), estes estudos não submeteram os participantes à USTC, SPECT com 99mTc-TRODAT e SST-16. Ao contrário desta tese, publicações anteriores não obtiveram curvas ROC, valores de corte e acurácia dos referidos métodos, tampouco verificaram se a acurácia da USTC combinada a SST-16 pode se comparar à acurácia do SPECT com 99mTc-TRODAT; o último é considerado método de referência para parkinsonismo de etiologia degenerativa devido a sua alta sensibilidade e especificidade (Huang et al., 2004, Shih et al., 2007; Wang et al., 2005; Wu et al., 2011). Na presente casuística, quando os resultados da USTC e SST-16 foram discordantes, SPECT com 99mTc-TRODAT auxiliou na confirmação da DP. Por outro lado, resultados concordantes entre USTC e SST-16 confirmaram os resultados do SPECT com 99mTC-TRODAT. Estes dados sugerem que resultados discordantes dos métodos USTC e 
SST-16 reforçam a indicação de SPECT com 99mTc-TRODAT, enquanto que resultados concordantes podem dispensar o uso de método invasivo. 0 ineditismo deste estudo consiste em comparar a acurácia da combinação de 2 métodos não invasivos (USTC e SST-16) com a acurácia de um método invasivo e radioativo (SPECT com TRODAT) para diagnóstico de DP. Protocolos anteriores não realizaram nos grupos casos e controles saudáveis a avaliação sonográfica, olfatória e molecular.

PET e SPECT para visualização dos TDA estriatais são considerados métodos de acurácia elevada para o diagnóstico da DP (Fallahi et al., 2016; Huang et al., 2004). Entretanto, são métodos caros, invasivos (necessitam infusão endovenosa de substâncias radioativas) e pouco disponíveis no mundo. PET e SPECT necessitam de equipamentos caros, salas amplas, isolamento à radiação, disponibilidade de radiotraçadores e equipe especializada (custo de manutenção elevado). Em contrapartida, USTC e SST-16 requerem somente salas ventiladas, equipamentos e materiais mais baratos e equipe especializada menor (custo de manutenção mais baixo).

Este estudo proveu resultados relevantes e potencialmente de impacto para prática clínica ao estabelecer uma nova estratégia de investigação diagnóstica para DP. A combinação de dois métodos diagnósticos não invasivos, mais baratos e facilmente disponíveis (USTC e SST-16) mostrou-se viável para pesquisa clínica e prática assistencial, inclusive em regiões desprovidas de recursos financeiros. Ainda, a combinação dos referidos métodos potencialmente possibilita a identificação de indivíduos em fase pré-clínica da DP - HSN e hiposmia são marcadores encontrados em fase pré-clínica da DP. 
HSN é reconhecida como marcador da DP (Berg et al., 1999; Berg, 2011b; Budsic et al., 2009). Na presente pesquisa, este sinal sonográfico foi encontrado em todos os doentes com DP e em torno de $22,2 \%$ dos indivíduos normais. A acurácia da USTC foi satisfatória para diferenciar indivíduos com DP dos participantes saudáveis. A USTC pode igualmente ser considerada ferramenta de acurácia satisfatória para diferenciar DP de outras condições (TE, parkinsonismo vascular e SPA) (Walter et al., 2004, 2007b; Budisic et al., 2009).

Em uma tese de livre-docência na FMUSP, Bor-Seng-Shu (2012) discutiu se diferentes equipamentos de ultrassonografia interferem na aferição da área ecogênica da $\mathrm{SN}$ e verificou que os valores de corte para identificar HSN são similares nas diversas populações, mesmo com emprego de diferentes marcas e modelos de equipamentos. O autor reforçou a importância de adotar as diretrizes da Sociedade Europeia de Neurossonologia e Hemodinâmica Cerebral para mensurar a área ecogênica da SN (Bor-SengShu, 2012).

Adotando PET para imagem dos TDA como referência, a USTC apresentou sensibilidade de $64,7 \%$ e especificidade de $60 \%$ para o diagnóstico precoce de DP (Li et al., 2015). Nesta casuística, USTC para avaliação ecogênica da SN apresentou valor preditivo positivo elevado, apesar de sensibilidade e especificidade moderadas, o que permitiu afirmar que resultados negativos não excluem o diagnóstico de DP e os resultados positivos (demonstração de $\mathrm{HSN}$ ) reduzem a necessidade de imagem molecular dos TDA (Liu et al., 2014). Ao contrário da maioria dos estudos prévios, incluindo o presente estudo, a sensibilidade e especificidade da USTC 
não foram altas; a alta incidência de hiperostose dos ossos temporais na população idosa asiática justifica esse achado.

Com respeito ao teste SST-16, o grupo DP apresentou redução marcante da função olfatória em relação ao grupo controle. Pesquisa que utilizou 12 itens do teste Sniffin' sticks demonstrou sensibilidade de $75 \%$ e especificidade de $70 \%$ para o diagnóstico de DP (Busse et al., 2012). Outro estudo aplicou o mesmo método de avaliação olfatória em brasileiros com DP (SST-16) e relatou valor da especificidade maior que o da sensibilidade para o diagnóstico de DP, respectivamente, $89 \%$ e $81 \%$ (Silveira-Moriyama et al., 2008).

A estratégia da combinação de USTC e testes de olfação para aumentar a acurácia do diagnóstico de DP tem sido reportado na literatura médica. Estudo recente que utilizou USTC e teste Sniffin' sticks de 12 itens relatou resultados anormais destes testes em $63 \%$ dos indivíduos com DP, $4,3 \%$ dos indivíduos normais e em nenhum dos pacientes com TE. Adicionalmente, a acurácia da USTC foi maior que a do teste de olfação, diferentemente do nosso estudo (Lopez-Hernández et al., 2015). Em um protocolo de atendimento que utilizou USTC, teste de olfação e cintilografia do miocárdio com ${ }^{123}$ I-metaiodobenzilguanidina, os autores verificaram que a combinação da USTC e teste de olfação (se um ou outro estivesse alterado) discriminou pacientes com DP dos controles saudáveis com maior sensibilidade, especificidade, valor preditivo positivo e negativo em relação a esses métodos se utilizados isoladamente (Izawa et al., 2012). Um estudo realizou USTC e teste de olfação em 715 participantes e revelou especificidade de $86,5 \%$ para USTC e $74,9 \%$ para teste de olfação, quando realizados 
isoladamente para o diagnóstico de DP. A combinação destes métodos aumentou significativamente a especificidade para $97,7 \%$; o valor preditivo positivo desta combinação triplicou, alcançando 17,6\% (valor preditivo positivo para USTC foi $5,2 \%$ e para teste de olfação, $2,5 \%$ ) (Tunc et al., 2015). Outro estudo que combinou USTC com teste de olfação também utilizou a variável assimetria motora, definida como assimetria na pontuação do UPDRS III, com intuito de diferenciar pacientes com DP de outras síndromes parkinsonianas. Contrariamente aos resultados apresentados, não houve diferença marcante entre USTC e teste de olfação em termos de sensibilidade e especificidade. A combinação de ambos aumentou a acurácia na diferenciação da DP de outras formas de parkinsonismo (Busse et al., 2012).

O presente estudo tem limitações: 1) amostra pequena, principalmente no grupo controle, em decorrência da dificuldade de recrutar voluntários sadios para exame invasivo e com exposição à radiação, 2) intervalo grande entre a realização do SPECT com TRODAT e USTC (apesar de a área ecogênica da SN manter-se constante com o tempo) (Berg et al., 2005) e 3) dificuldade de acobertamento (cegamento) completo dos examinadores da USTC e teste da olfação em relação a sinais clínicos de parkinsonismo. 
CONCLUSÕES 
O presente estudo permite concluir:

1) A acurácia da combinação da ultrassonografia transcraniana com teste de olfação é satisfatória e maior em comparação a acurácia dos referidos métodos isoladamente para o diagnóstico da Doença de Parkinson.

2) A acurácia do método combinado (Ultrassonografia transcraniana e teste de olfação) aproximou-se à acurácia de SPECT com TRODAT para detectar indivíduos com doença de Parkinson. 
ANEXOS 
Anexo A - United Kingdom Parkinson`s Disease Society Brain Bank Clinical Diagnostic Criteria (GIBB, LEE, 1988)

Passo 1 . Diagnóstico de síndrome parkinsoniana: Bradicinesia e pelo menos 1 dos seguintes ítens:
a) rigidez
b) tremor de repouso $(4-6 \mathrm{~Hz})$
c) instabilidade postural

Passo 2. Critérios de exclusão da DP: Acidente vascular encefálico de repetição; trauma crânioencefálico, encefalite; crise oculogírica; uso do neurolépticos quando do início dos sintomas; presença de mais de 1 parente afetado; remissão sustentada; doença estritamente unilateral até 3 anos; paralisia do olhar vertical; sinais cerebelares; envolvimento autonômico precoce intenso; demência de início precoce ou anormalidade precoce da linguagem, memória e praxia; sinal de Babinski; tumor encefálico ou hidrocefalia comunicante; resposta negativa a altas doses de levodopa; exposição a 1metil-4-fenil-1,2,3,6-tetraidropiridina.

Passo 3. Critérios prospectivos e positivos de suporte ao diagnóstico de DP (3 ou mais são necessários para o diagnóstico definitivo)

Início unilateral; tremor de repouso presente; doença progressiva; assimetria persistente afetando o lado do início dos sintomas com excelente resposta a levodopa (70 a 100\%); coréia grave induzida por levodopa; resposta a levodopa por mais de 5 anos e curso da doença de 10 anos ou mais. 
Anexo B - Unified Rating Parkinson`s Disease Rating Scale - Parte III

\section{EXAME MOTOR}

- fala

$0=$ normal.

$1=$ perda discreta da expressão, volume ou dicção.

2=comprometimento moderado. Arrastado, monótono mas compreensível.

3= comprometimento grave, difícil de ser entendido.

4= incompreensível.

- expressão facial

$0=$ normal.

1= hipomimia mínima.

2= diminuição pequena, mas anormal, da expressão facial.

3= hipomimia moderada, lábios caídos/afastados por algum tempo.

4= fácies em máscara ou fixa, com perda grave ou total da expressão facial. Lábios afastados em $1 / 4$ de polegada ou mais.

- tremor de repouso: face, lábios, queixo 14

Mão direita 14; mão esquerda 14; Pé direito 14; Pé esquerdo 14 $0=$ ausente

1= presente, mas infrequente ou leve.

2= persistente, mas de pouca amplitude; ou moderado em amplitude mas presente de maneira intermitente.

3= moderado em amplitude mas presente a maior parte do tempo.

4= com grande amplitude e presente a maior parte do tempo. 
- tremor postural ou de ação nas mãos: Mão direita 14; mão esquerda 14

$0=$ ausente

$1=$ leve, presente com a ação.

2= moderado em amplitude, presente com a ação.

3= moderado em amplitude tanto na ação quanto mantendo a postura.

4= grande amplitude, interferindo com a alimentação.

- rigidez (movimento passivo das grandes articulações, com paciente sentado e relaxado, ignorar roda denteada)

Pescoço ___ 14; Membro superior direito ___ 14 ; esquerdo ___ 14

Membro inferior direito ___ 14 ; esquerdo ___ 14

$0=$ ausente

1= pequena ou detectável somente quando ativado por movimentos em espelho de outros.

2= leve e moderado.

3= marcante, mas pode realizar o movimento completo da articulação.

4= grave e o movimento completo da articulação só ocorre com grande dificuldade.

- bater dedos continuamente - polegar no indicador em seqüências rápidas com a maior amplitude possível, uma mão de cada vez: Mão direita 14 mão esquerda ___ 14

$0=$ normal

1= leve lentidão e/ou redução da amplitude. 
2= comprometimento moderado. Fadiga precoce e bem clara. Pode apresentar parada ocasional durante o movimento.

3= comprometimento grave. Hesitação freqüente para iniciar o movimento ou paradas durante o movimento que está realizando.

4= realiza o teste com grande dificuldade, quase não conseguindo.

- movimentos das mãos (abrir e fechar as mãos em movimentos rápidos e sucessivos e com a maior amplitude possível, uma mão de cada vez).

Mão direita 14; mão esquerda 14

$0=$ normal

1= leve lentidão e/ou redução da amplitude.

2= comprometimento moderado. Fadiga precoce e bem clara. Pode apresentar parada ocasional durante o movimento.

3= comprometimento grave. Hesitação freqüente para iniciar o movimento ou paradas durante o movimento que está realizando.

4= realiza o teste com grande dificuldade, quase não conseguindo.

- movimentos rápidos alternados das mãos (pronação e supinação das mãos, horizontal ou verticalmente, com a maior amplitude possível, as duas mãos simultaneamente).

Mão direita 14; mão esquerda 14

$0=$ normal

1= leve lentidão e/ou redução da amplitude.

2= comprometimento moderado. Fadiga precoce e bem clara. Pode apresentar parada ocasional durante o movimento. 
3= comprometimento grave. Hesitação freqüente para iniciar o movimento ou paradas durante o movimento que está realizando.

4= realiza o teste com grande dificuldade, quase não conseguindo.

- agilidade da perna (bater o calcanhar no chão em sucessões rápidas, levantando toda a perna, a amplitude do movimento deve ser de cerca de 3 polegadas/ $\pm 7,5 \mathrm{~cm})$. Perna Direita 14; perna esquerda 14

$0=$ normal

1= leve lentidão e/ou redução da amplitude.

2= comprometimento moderado. Fadiga precoce e bem clara. Pode apresentar parada ocasional durante o movimento.

3= comprometimento grave. Hesitação freqüente para iniciar o movimento ou paradas durante o movimento que está realizando.

4= realiza o teste com grande dificuldade, quase não conseguindo.

- levantar da cadeira (de espaldo reto, madeira ou ferro, com braços cruzados em frente ao peito).

$0=$ normal

$1=$ lento ou pode precisar de mais de uma tentativa

2= levanta-se apoiando nos braços da cadeira .

$3=$ tende a cair para trás, pode tentar se levantar mais de uma vez, mas consegue levantar

4= incapaz de levantar-se sem ajuda. 
- postura

$0=$ normal em posição ereta.

1= não bem ereto, levemente curvado para frente, pode ser normal para pessoas mais velhas.

2= moderadamente curvado para frente, definitivamente anormal, pode inclinarse um pouco para os lados.

3= acentuadamente curvado para frente com cifose, inclinação moderada para um dos lados.

4= bem fletido com anormalidade acentuada da postura.

- marcha

$0=$ normal

$1=$ anda lentamente, pode arrastar os pés com pequenas passadas, mas não há festinação ou propulsão.

2= anda com dificuldade, mas precisa de pouca ajuda ou nenhuma, pode apresentar alguma festinação, passos curtos, ou propulsão.

$3=$ comprometimento grave da marcha, necessitando de ajuda.

4= não consegue andar sozinho, mesmo com ajuda.

- estabilidade postural (respostas ao deslocamento súbito para trás, puxando os ombros, com paciente ereto, de olhos abertos, pés separados, informado a respeito do teste)

$0=$ normal

1= retropulsão, mas se recupera sem ajuda. 
2= ausência de respostas posturais, cairia se não fosse auxiliado pelo examinador.

3= muito instável, perde o equilíbrio espontaneamente.

4= incapaz de ficar ereto sem ajuda.

- bradicinesia e hipocinesia corporal (combinação de hesitação, diminuição do balançar dos braços, pobreza e pequena amplitude de movimentos em geral) $0=$ nenhum.

1= lentidão mínima. Podia ser normal em algumas pessoas. Possível redução na amplitude.

2= movimento definitivamente anormal. Pobreza de movimento e um certo grau de lentidão.

3= lentidão moderada. Pobreza de movimento ou com pequena amplitude.

4= lentidão acentuada. Pobreza de movimento ou com pequena amplitude. 
Anexo C - Escala de Hoenh and Yahr modificada (HOEHN, YARHR, 1976; SHENKMAN et al., 2001)

ESTÁGIO 0 - Nenhum sinal da doença

ESTÁGIO 1 - Doença unilateral

ESTÁGIO 1,5 - Envolvimento unilateral e axial

ESTÁGIO 2 - Doença bilateral sem déficit de equilíbrio

ESTÁGIO 2,5 - Doença bilateral leve, com recuperação no "teste do empurrão"

ESTÁGIO 3 - Doença bilateral leve a moderada; alguma instabilidade postural; capacidade para viver independente

ESTÁGIO 4 - Incapacidade grave, ainda capaz de caminhar ou permanecer de pé sem ajuda

ESTÁGIO 5 - Confinado à cama ou cadeira de rodas a não ser que receba ajuda. 
Anexo D - Escala de Schwab and England (SCHWAB, ENGLAND, 1969)

$100 \%$ - Completemente independente. Hábil para fazer todas tarefas sem lentificação, dificuldade ou prejuízo

90\% - Completamente independente. Hábil para fazer todas tarefas com alguma lentificação, dificuldade ou prejuízo. Pode levar o tempo dobrado

$\mathbf{8 0 \%}$ - Independente na maioria das tarefas. Leva o tempo dobrado para realizá-las. Consciente da dificuldade e lentificação

$70 \%$ - Não completamente independente. Mais dificuldades com algumas tarefas. 3 a 4 vezes mais tempo em algumas tarefas. Pode levar grande parte do tempo para algumas atividades

$60 \%$ - Alguma dependência. Pode fazer a maioria das tarefas, mas muito lento e com muito esforço, erros, algumas impossíveis

$\mathbf{5 0 \%}$ - Mais dependente. Ajuda com metade das tarefas. Dificuldade com tudo 40\% - Muito dependente. Pode precisar de assistência em todas as tarefas, mas faz algumas sozinho

$30 \%$ - Com algum esforço, consegue fazer algumas atividades sozinho. Muita ajuda é necessária

$20 \%$ - Não faz atividades sozinho. Pode com discreta ajuda, fazer algumas atividades sozinho. Muito inválido

$10 \%$ - Totalmente dependente

0\% - Funções vegetativas como deglutição, função vesical e intestinal não estão funcionando. Restrito a cama 
Anexo E - Miniexame do Estado Mental (FOLSTEIN, FOLSTEIN, MCHUGH, 1975)

\section{Orientação}

1) Dia da Semana (1 ponto)

2) Dia do Mês (1 ponto)

3) Mês (1 ponto)

4) Ano (1 ponto)

5) Hora aproximada (1 ponto)

6) Local específico (andar ou setor) (1 ponto

7) Instituição (residência, hospital, clínica) (1 ponto

8) Bairro ou rua próxima (1 ponto)

9) Cidade (1 ponto)

10)Estado (1 ponto)

\section{Memória Imediata}

Fale três palavras não relacionadas. Posteriormente pergunte ao paciente pelas 3 palavras. Dê 1 ponto para cada resposta correta.

Depois repita as palavras e certifique-se de que o paciente as aprendeu, pois mais adiante você irá perguntá-las novamente.

\section{Atenção e Cálculo}

(100-7) sucessivos, 5 vezes sucessivamente $(93,86,79,72,65)$

(1 ponto para cada cálculo correto)

\section{Evocação}

Pergunte pelas três palavras ditas anteriormente (1 ponto por palavra)

\section{Linguagem}

1) Nomear um relógio e uma caneta (2 pontos)

2) Repetir "nem aqui, nem ali, nem lá" (1 ponto)

3) Comando:"pegue este papel com a mão direita, dobre ao meio e coloque no chão (3 pontos)

4) Ler e obedecer:"feche os olhos" (1 ponto)

5) Escrever uma frase (1 ponto)

6) Copiar um desenho (1 ponto)

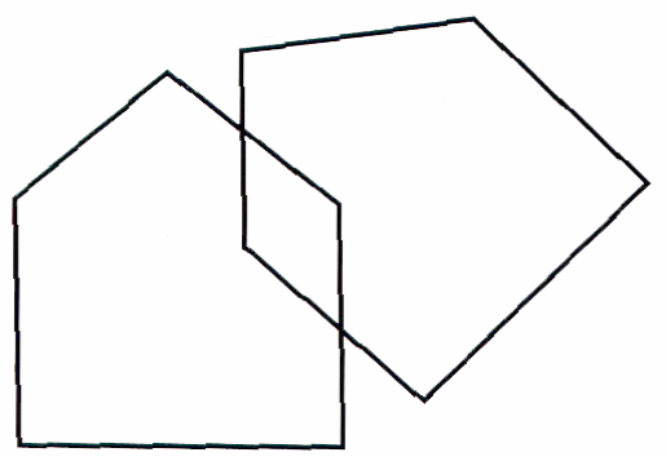

Escore: ( 
Anexo F - Inventário de depressão de Beck (GORESTEIN, ANDRADE, 1996)

Neste questionário existem grupos de afirmativas. Por favor leia cada uma delas e selecione a afirmativa que melhor descreva como você se sentiu NA SEMANA QUE PASSOU, INCLUINDO O DIA DE HOJE. Desenhe um círculo ao lado da afirmativa que tiver selecionado.

Se várias afirmativas no grupo parecem aplicar-se igualmente bem, circule cada uma delas.

Certifique-se de ter lido todas as afirmativas antes de fazer sua escolha.

1) 0 Não me sinto triste.

I Sinto-me triste.

2 Sinto-me triste o tempo todo e não consigo sair disso.

3 Estou tão triste e infeliz que não posso agüentar.

2) 0 Não estou particularmente desencorajado quanto ao futuro.

I Sinto-me desencorajado quanto ao futuro.

2 Sinto que não tenho nada por que esperar.

3 Sinto que o futuro é sem esperança e que as coisas não podem melhorar.

3) 0 Não me sinto fracassado.

I Sinto que falhei mais do que o indivíduo médio.

2 Quando olho para trás em minha vida, tudo que vejo é uma porção de fracassos. 
3 Sinto que sou um fracasso completo como pessoa.

4) 0 Obtenho tanta satisfação com as coisas como costumava fazer.

I Não gosto das coisas da maneira como costumava gostar.

2 Não consigo mais sentir satisfação real com coisa alguma.

3 Estou insatisfeito ou entediado o tempo todo.

5) 0 Não me sinto particularmente culpado.

I Sinto-me culpado boa parte do tempo.

2 Sinto-me muito culpado a maior parte do tempo.

3 Sinto-me culpado o tempo todo.

6) 0 Não sinto que esteja sendo punido.

1 Sinto que posso ser punido.

2 Sinto que estou sendo punido.

3 Sinto que estou a ser punido(a).

7) 0 Não me sinto desapontado comigo mesmo.

I Sinto-me desapontado comigo mesmo.

2 Sinto-me aborrecido comigo mesmo.

3 Eu me odeio.

8) 0 Não sinto que seja pior que qualquer outra pessoa. 
I Critico minhas fraquezas ou erros.

2 Responsabilizo-me o tempo todo por minhas falhas.

3 Culpo-me por todas as coisas ruins que acontecem.

9) 0 Não tenho nenhum pensamento a respeito de matar.

I Tenho pensamentos sobre me matar mas não os levaria adiante.

2 Gostaria de matar.

3 Eu me mataria se tivesse uma oportunidade.

10) 0 Não costumo chorar mais que o habitual.

I Choro mais agora do que costumava fazer.

2 Atualmente choro o tempo todo.

3 Eu costumava conseguir chorar, mas agora não consigo, mesmo que queira.

11) 0 Não me irrito mais agora que em qualquer outra época.

I Fico molestado ou irritado mais facilmente do que costumava.

2 Atualmente sinto-me irritado todo tempo.

3 Absolutamente não me irrito com as coisas que costumavam irritarme.

12) 0 Não perdi o interesse nas outras pessoas.

I Interesso-me menos do que costumava pelas outras pessoas.

2 Perdi a maior parte do meu interesse nas outras pessoas.

3 Perdi todo o meu interesse nas outras pessoas. 
13) 0 Tomo decisões mais ou menos tão bem como em qualquer outra época.

I Adio minhas decisões mais do que costumava.

2 Tenho maior dificuldade em tomar decisões mais do que antes.

3 Não consigo mais tomar decisões.

14) 0 Não sinto que minha aparência seja pior do que costumava ser.

I Preocupo-me por estar parecendo velho ou sem atrativos.

2 Sinto mudanças permanentes em minha aparência que me fazem parecer sem atrativos.

3 Considero-me feio.

15) 0 Posso trabalhar mais ou menos tão bem quanto antes.

I Preciso de um esforço extra para começar qualquer coisa.

2 Tenho que me forçar muito até fazer qualquer coisa.

3 Não consigo fazer nenhum trabalho.

16) Durmo tão bem quanto de hábito.

I Não durmo tão bem quanto costumava.

2 Acordo 1 ou 2 horas mais cedo do que de hábito e tenho dificuldade de voltar a dormir.

3 Acordo várias horas mais cedo do que costumava e tenho dificuldade de voltar a dormir. 
17) 0 Não fico mais cansado que o hábito.

I Fico cansado com mais facilidade do que costumava.

2 Sinto-me cansado ao fazer quase qualquer coisa.

3 Estou cansado demais para fazer qualquer coisa.

18) 0 Meu apetite não está pior do que de hábito.

I Meu apetite não é tão bom como costumava ser.

2 Meu apetite está muito pior agora.

3 Não tenho mais nenhum apetite.

19) 0 Não perdi muito peso se é que perdi algum ultimamente.

I Perdi mais de $2,5 \mathrm{Kg}$.

2 Perdi mais de $5,0 \mathrm{Kg}$

3 Perdi mais de 7,5

Estou deliberadamente tentando perder peso, comendo menos:( ) Sim ( ) Não.

20) 0 Não me preocupo mais de hábito com minha saúde.

I Preocupo-me com problemas físicos, como dores e aflições no estômago ou prisões de ventre.

2 Estou muito preocupado com problemas físicos e é difícil pensar em muito mais que isso.

3 Estou tão preocupado com meus problemas físicos que não consigo pensar em outra coisa. 
21) 0 Não tenho observado qualquer mudança recente em meu interesse sexual.

I Estou menos interessado por sexo do que costumava.

2 Estou bem menos interessado em sexo atualmente.

3 Perdi completamente o interesse por sexo. 
Anexo G - Comprovante de aprovação no Comitê de ética em pesquisa

\author{
Hospital das Clínicas da FMUSP \\ Comissäo de Ética para Análise de Projetos de Pesquisa \\ CAPPesq
}

No Protocolo: 0812/10

Título: "Ultrassonografia Transcraniana Do Parēnquima Encetálico Nos Distúrbios: De Movimento"

Pesquisador Responsável: Dr. Edson Bor-Seng-Shu / Prof.Dr. Manoel Jacobsen Teixeira

Pesquisador Executante: Dr. Edson Bor-Seng-Shu

Departamento: Neurologia

A Comissõo de Ética para Análise de Projetos de Pesquisa-CAPPesa da Diretoria Clinica do Hospital das Clinicas da Faculdade de Medicina da Universidade de Säo Paulo, Aprovou / TOMOU CIÊNCIA na sessâo datada de 23/08/2011, do(s) documento(s) abaixo mencionado(s):

- Carta datada de 27/07/11 - S olicitaçōo de inclusāo dos pesquisadores executantes Miguel César Merino Ruiz, Kelson James Silva de Almeida e Egberto Reis Barbosa e Mudança de titulo para: "Ultrassonografia transcraniana na doença de Parkinson"

A CAPPesq em obediência à Resolução CNS 196/96, solicita ao pesquisador (a) s elaboração de relatorio parcial e final.

No caso de relatório parcial é necessário informar o tempo previsto para a conclusâo do protocolo e breve resumo dos resultados obtidos.

CAPPesq, 23 de Agosto de 2011

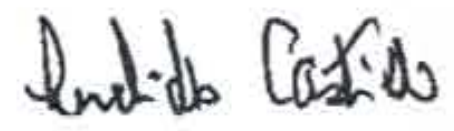

PROF. DR. EUCLIDES AYRES DE CASTILHO

Coordenador

Comissão de Ética para Análise de

Projetos de Pesquisa - CAPPesq 
REFERÊNCIAS 
Ansari KA, Johnson A. Olfactory function in patients with Parkinson's disease. J Chronic Dis. 1975; 28: 493-7.

Associação Brasileira de normas técnicas. NBR 6023: informação e documentação: referências: elaboração. Rio de Janeiro (RJ); 2002.

Barbosa MT, Caramelli P, Maia DP, Cunningham MC, Guerra HL, Lima-Costa MF, Cardoso F. Parkinsonism and Parkinson's disease in the elderly: a community-based survey in Brazil (the Bambui study). Mov Disord. 2006; 21: 800-8.

Barone P, Antonini A, Colosimo C, Marconi R, Morgante L, Avarello TP, Bottacchi E, Cannas A, Ceravolo G, Ceravolo R, Cicarelli G, Gaglio RM, Giglia RM, lemolo F, Manfredi M, Meco G, Nicoletti A, Pederzoli M, Petrone A, Pisani A, Pontieri FE, Quatrale R, Ramat S, Scala R, Volpe G, Zappulla S, Bentivoglio AR, Stocchi F, Trianni G, Dotto PD; PRIAMO study group. The PRIAMO study: a multicenter assessment of nonmotor symptoms and their impact on quality of life in Parkinson's disease. Mov Disord. 2009; 24: 1641-9.

Bártová $\mathrm{P}$, Skoloudík D, Ressner $\mathrm{P}$, Langová $\mathrm{K}$, Herzig $\mathrm{R}$, Kanovsky $\mathrm{P}$. Correlation between substantia nigra features detected by sonography and Parkinson disease symptoms. J Ultrasound Med. 2010; 29: 37-42. 
Becker G, Seufert J, Bogdahn U, Reichmann H, Reiners K. Degeneration of substantia nigra in chronic Parkinson's disease visualized by transcranial colorcoded real-time sonography. Neurology. 1995; 45:182-4.

Berg D, Becker G, Zeiler B, Tucha O, Hofmann E, Preier M, Benz P, Jost W, Reiners K, Lange KW. Vulnerability of the nigrostriatal system as detected by transcranial ultrasound. Neurology. 1999; 53:1026-31.

Berg D, Jabs B, Merschdorf U, Beckmann H, Becker G. Echogenicity of substantia nigra determined by transcranial ultrasound correlates with severity of parkinsonian symptoms induced by neuroleptic therapy. Biol Psychiatry. 2001a; 50: 463-7.

Berg D, Siefker C, Becker G. Echogenicity of the substantia nigra in Parkinson's disease and its relation to clinical findings. J Neurol. 2001b; 248:684-9.

Berg D, Merz B, Reiners K, Naumann M, Becker G. Five-year follow-up study of hyperechogenicity of the substantia nigra in Parkinson's disease. Mov Disord. $2005 ; 20(3): 383-5$.

Berg D. Substantia nigra hyperechogenicity is a risk marker of Parkinson's disease: yes. J Neural Transm (Vienna). 2011a; 118: 613-9.

Berg D, Seppi K, Behnke S, Liepelt I, Schweitzer K, Stockner H, Wollenweber F, Gaenslen A, Mahlknecht P, Spiegel J, Godau J, Huber H, Srulijes K, Kiechl S, 
Bentele M, Gasperi A, Schubert T, Hiry T, Probst M, Schneider V, Klenk J, Sawires M, Willeit J, Maetzler W, Fassbender K, Gasser T, Poewe W. Enlarged substantia nigra hyperechogenicity and risk for Parkinson disease: a 37-month 3-center study of 1847 older persons. Arch Neurol. 2011b; 68: 932-7.

Berg D, Godau J, Seppi K, Behnke S, Liepelt-Scarfone I, Lerche S, Stockner H, Gaenslen A, Mahlknecht P, Huber H, Srulijes K, Klenk J, Fassbender K, Maetzler W, Poewe W; PRIPS study group. The PRIPS study: screening battery for subjects at risk for Parkinson's disease. Eur J Neurol. 2013; 20: 102-8.

Bonnet AM, Jutras MF, Czernecki V, Corvol JC, Vidailhet M. Nonmotor Symptoms in Parkinson's Disease in 2012: Relevant Clinical Aspects. Parkinsons Dis. 2012; 2012: 198316.

Bor-Seng-Shu E, Fonoff ET, Barbosa ER, Teixeira MJ. Substantia nigra hyperechogenicity in Parkinson`s disease. Acta Neurochir (Wien). 2010; 152: 2085-7.

Bor-Seng-Shu E. Doença de Parkinson: ecogenicidade da substância negra e imagem molecular dos transportadores de dopamina. [tese livre-docência]. São Paulo: Faculdade de Medicina, Universidade de São Paulo; 2012.

Bor-Seng-Shu E, Almeida KJ, Andrade DC, Fonoff ET, Teixeira MJ, Barbosa ER. Echogenicity of the Substantia nigra region in Parkinson`s disease. Arq Neuropsiquiatr. 2012; 70: 153-4. 
Bor-Seng-Shu E, Pedroso JL, Felicio AC, de Andrade DC, Teixeira MJ, BragaNeto P, Batista IR, Barsottini OG, Borges V, Ferraz HB, Shih MC, Bressan RA, de Andrade LA, Walter U. Substantia nigra echogenicity and imaging of striatal dopamine transporters in Parkinson's disease: A cross-sectional study. Parkinsonism Relat Disord. 2014a; 20: 477-81.

Bor-Seng-Shu E, Felicio AC, Braga-Neto P, Batista IR, Paiva WS, de Andrade DC, Teixeira MJ, de Andrade LA, Barsottini OG, Shih MC, Bressan RA, Ferraz HB. Dopamine Transporter Imaging Using 99mTc-TRODAT-1 SPECT in Parkinson's Disease. Med Sci Monit. 2014b; 20: 1413-8.

Boulton AA, Eisenhofer G. Catecholamine metabolism. From molecular understanding to clinical diagnosis and treatment. Overview. Adv Pharmacol. 1998; 42: 273-92.

Bouwmans AE, Vlaar AM, Mess WH, Kessels A, Weber WE. Specificity and sensitivity of transcranial sonography of the substantia nigra in the diagnosis of Parkinson's disease: prospective cohort study in 196 patients. BMJ Open. 2013; 3: 2613.

Braak H, Del Tredici K, Rüb U, de Vos RA, Jansen Steur EN, Braak E. Staging of brain pathology related to sporadic Parkinson's disease. Neurobiol Aging. 2003; 24: 197-211. 
Braak H, Bohl JR, Müller CM, Rüb U, de Vos RA, Del Tredici K. Stanley Fahn Lecture 2005: The staging procedure for the inclusion body pathology associated with sporadic Parkinson's disease reconsidered. Mov Disord. 2006; 21: $2042-51$

Brucki SM, Nitrini R, Caramelli P, Bertolucci PH, Okamoto IH. Suggestions for utilization of the mini-mental state examination in Brazil. Arq Neuropsiquiatr. 2003; 61: 777-81.

Budisic M, Trkanjec Z, Bosnjak J, Lovrencic-Huzjan A, Vukovic V, Demarin V. Distinguishing Parkinson's disease and essential tremor with transcranial sonography. Acta Neurol Scand. 2009; 119: 17-21.

Busse K, Heilmann R, Kleinschmidt S, Abu-Mugheisib M, Höppner J, Wunderlich C, Gemende I, Kaulitz L, Wolters A, Benecke R, Walter U. Value of combined midbrain sonography, olfactory and motor function assessment in the differential diagnosis of early Parkinson's disease. J Neurol Neurosurg Psychiatry. 2012; 83: 441-7.

Chang LT. A method for attenuation correction in radionuclide computed tomopraphy. IEEE Trans Nucl Sci. 1978; 25: 638-43.

Cosentino C, Nuñez Y, Torres L. Frequency of non-motor symptoms in Peruvian patients with Parkinson's disease. Arq Neuropsiquiatr. 2013; 71: 216-9. 
De Rijk MC, Launer LJ, Berger K, Breteler MM, Dartigues JF, Baldereschi M, Fratiglioni L, Lobo A, Martinez-Lage J, Trenkwalder C, Hofman A. Prevalence of Parkinson's disease in Europe: A collaborative study of population-based cohorts. Neurologic Diseases in the Elderly Research Group. Neurology. 2000; 54: S21-3.

Doepp F, Plotkin M, Siegel L, Kivi A, Gruber D, Lobsien E, Kupsch A, Schreiber SJ. Brain parenchyma sonography and 123I-FP-CIT SPECT in Parkinson's disease and essential tremor. Mov Disord. 2008; 23: 405-10.

Fallahi B, Esmaeili A, Beiki D, Oveisgharan S, Noorollahi-Moghaddam H, Erfani M, Tafakhori A, Rohani M, Fard-Esfahani A, Emami-Ardekani A, Geramifar P, Eftekhari M. Evaluation of 99mTc-TRODAT-1 SPECT in the diagnosis of Parkinson's disease versus other progressive movement disorders. Ann Nucl Med. 2016; 30: 153-62.

Felicio AC, Moriyama TS, Godeiro-Junior C, Shih MC, Hoexter MQ, Borges V, Silva SM, Amaro-Junior E, Andrade LA, Ferraz HB, Bressan RA. Higher dopamine transporter density in Parkinson's disease patients with depression. Psychopharmacology (Berl). 2010; 211: 27-31.

Fereshtehnejad SM, Romenets SR, Anang JB, Latreille V, Gagnon JF, Postuma RB. New clinical subtypes of parkinson disease and their longitudinal progression: a prospective cohort comparison with other phenotypes. JAMA Neurol. 2015; 72: 863-73. 
Folstein MF, Folstein SE, McHugh PR. "Mini-Mental State". A practical method for grading the cognitive state of patients for the clinician. J Psychiatr Res. 1975; 12: 189-98.

Gaenslen A, Unmuth B, Godau J, Liepelt I, Di Santo A, Schweitzer KJ, Gasser T, Machulla HJ, Reimold M, Marek K, Berg D. The specificity and sensitivity of transcranial ultrasound in the differential diagnosis of Parkinson's disease: a prospective blinded study. Lancet Neurol. 2008; 7: 417-24.

Gibb WR, Lees AJ. The relevance of the Lewy body to the pathogenesis of idiopathic Parkinson`s disease. J Neurol Neurosurg Psychiatry. 1988; 51: $745-52$.

Gorenstein C, Andrade L. Validation of a Portuguese version of the Beck Depression Inventory and the State-Trait Anxiety Inventory in Brazilian subjects. Braz J Med Biol Res. 1996; 29: 453-7.

Haehner A, Hummel T, Hummel C, Sommer U, Junghanns S, Reichmann H. Olfactory loss may be a first sign of idiopathic Parkinson's disease. Mov Disord. 2007; 22: 839-42.

Hoehn MM, Yahr MD. Parkinsonism: onset, progression and mortality. Neurology. 1967; 17: 427-42. 
Huang WS, Lee MS, Lin JC, Chen CY, Yang YW, Lin SZ, Wey SP. Usefulness of brain ${ }^{99 m}$ Tc-TRODAT-1 SPET for the evaluation of Parkinson's disease. Eur $\mathrm{J}$ Nucl Med Mol Imaging. 2004; 31: 155-61.

Hughes AJ, Daniel SE, Kilford L, Lees AJ. Accuracy of clinical diagnosis of idiopathic Parkinson's disease: a clinico-pathological study of 100 cases. J Neurol Neurosurg Psychiatry. 1992; 55: 181-4.

Hughes AJ, Daniel SE, Ben-Shlomo Y, Lees AJ. The accuracy of diagnosis of parkinsonian syndromes in a specialist movement disorders service. Brain. 2002; 125: 861-70.

Hüttenbrink KB, Hummel T, Berg D, Gasser T, Hähner A. Olfactory dysfunction: common in later life and early warning of neurodegenerative disease. Dtsch Arztebl Int. 2013; 110: 1-7.

Iranzo A, Lomeña $F$, Stockner $H$, Valldeoriola $F$, Vilaseca I, Salamero $M$, Molinuevo JL, Serradell M, Duch J, Pavía J, Gallego J, Seppi K, Högl B, Tolosa E, Poewe W, Santamaria J; Sleep Innsbruck Barcelona (SINBAR) group. Decreased striatal dopamine transporter uptake and substantia nigra hyperechogenicity as risk markers of synucleinopathy in patients with idiopathic rapid-eye-movement sleep behaviour disorder: a prospective study. Lancet Neurol. 2010; 9: 1070-7. 
Iranzo A, Stockner H, Serradell M, Seppi K, Valldeoriola F, Frauscher B, Molinuevo JL, Vilaseca I, Mitterling T, Gaig C, Vilas D, Santamaria J, Högl B, Tolosa E, Poewe W. Five-year follow-up of substantia nigra echogenicity in idiopathic REM sleep behavior disorder. Mov Disord. 2014; 29: 1774-80.

Izawa MO, Miwa $\mathrm{H}$, Kajimoto $\mathrm{Y}$, Kondo $\mathrm{T}$. Combination of transcranial sonography, olfactory testing, and MIBG myocardial scintigraphy as a diagnostic indicator for Parkinson's disease. Eur J Neurol. 2012; 19: 411-6.

Jankovic J. Parkinson's disease: clinical features and diagnosis. J Neurol Neurosurg Psychiatry. 2008; 79: 368-76.

Lauretani F, Saginario A, Ceda GP, Galuppo L, Ruffini L, Nardelli A, Maggio M. Treatment of the motor and non-motor symptoms in Parkinson's disease according to cluster symptoms presentation. Curr Drug Targets. 2014; 15: 943-7.

Lerche S, Seppi K, Behnke S, Liepelt-Scarfone I, Godau J, Mahlknecht P, Gaenslen A, Brockmann K, Srulijes K, Huber H, Wurster I, Stockner H, Kiechl S, Willeit J, Gasperi A, Fassbender K, Poewe W, Berg D. Risk factors and prodromal markers and the development of Parkinson's disease. J Neurol. $2014 ; 261: 180-7$.

Li DH, Zhang LY, Hu YY, Jiang XF, Zhou HY, Yang Q, Kang WY, Liu J, Chen SD. Transcranial sonography of the substantia nigra and its correlation with DAT- 
SPECT in the diagnosis of Parkinson's disease. Parkinsonism Relat Disord. 2015; 21: 923-8.

Liu P, Li X, Li FF, Ou-Yang QH, Zhang HX, Feng T. The predictive value of transcranial sonography in clinically diagnosed patients with early stage Parkinson's disease: comparison with DAT PET scans. Neurosci Lett. 2014; 582: 99-103.

López-Hernández N, García-Escrivá A, Shalabi-Benavent M. Diagnostic value of combined assessment of olfaction and substantia nigra hyperechogenicity for Parkinson's disease. Neurologia. 2015; 30: 496-501.

Miyamoto M, Miyamoto T. Neuroimaging of rapid eye movement sleep behavior disorder: transcranial ultrasound, single-photon emission computed tomography, and positron emission tomography scan data. Sleep Med. 2013; 14: $739-43$.

Morrison BM, Hof PR, Morrison JH. Determinants of neuronal vulnerability in neurodegenerative diseases. Ann Neurol. 1998; 44: S32-44.

O'Sullivan SS, Williams DR, Gallagher DA, Massey LA, Silveira-Moriyama L, Lees AJ. Nonmotor symptoms as presenting complaints in Parkinson's disease: a clinicopathological study. Mov Disord. 2008; 23: 101-6. 
Piccini PP. Dopamine transporter: basic aspects and neuroimaging. Mov Disord. 2003; 18: S3-8.

Pirker W, Asenbaum S, Bencsits G, Prayer D, Gerschlager W, Deecke L, Brücke T. [123I]beta-CIT SPECT in multiple system atrophy, progressive supranuclear palsy, and corticobasal degeneration. Mov Disord. 2000;15: 1158-67.

Politis M. Neuroimaging in Parkinson disease: from research setting to clinical practice. Nat Rev Neurol. 2014; 10: 708-22.

Ponsen MM, Stoffers D, Booij J, van Eck-Smit BL, Wolters ECh, Berendse HW. Idiopathic hyposmia as a preclinical sign of Parkinson's disease. Ann Neurol. 2004; 56: 173-81.

Ressner P, Skoloudík D, Hlustík P, Kanovský P. Hyperechogenicity of the substantia nigra in Parkinson's disease. J Neuroimaging. 2007; 17: 164-7.

Ross GW, Petrovitch H, Abbott RD, Tanner CM, Popper J, Masaki K, Launer L, White LR. Association of olfactory dysfunction with risk for future Parkinson's disease. Ann Neurol. 2008; 63: 167-73.

Santin R, Fonseca VF, Bleil CB, Rieder CR, Hilbig A. Olfactory function and Parkinson's disease in Southern Brazil. Arq Neuropsiquiatr. 2010; 68: 252-7. 
Schenkman ML, Clark K, Xie T, Kuchibhatla M, Shinberg M, Ray L. Spinal movement and performance of standing reach task in participants with and without Parkinson disease. Phys Ther. 2001; 81: 1400-11.

Scherfler C, Esterhammer R, Nocker M, Mahlknecht P, Stockner H, Warwitz B, Spielberger S, Pinter B, Donnemiller E, Decristoforo C, Virgolini I, Schocke M, Poewe W, Seppi K. Correlation of dopaminergic terminal dysfunction and microstructural abnormalities of the basal ganglia and the olfactory tract in Parkinson's disease. Brain. 2013; 136: 3028-37.

Schwab JF, England AC. Projection technique for evaluating surgery in Parkinson's disease. In: Billingham FH, Donaldson MC, editors. Third symposium on Parkinson's disease. Edinburgh: Livingstone, 1969; 152-157.

Seibyl JP. Imaging studies in movement disorders. Semin Nucl Med. 2003; 33: 105-13.

Shih MC, Franco de Andrade LA, Amaro E Jr, Felicio AC, Ferraz HB, Wagner J, Hoexter MQ, Lin LF, Fu YK, Mari JJ, Tufik S, Bressan RA. Higher nigrostriatal dopamine neuron loss in early than late onset Parkinson's disease? - a [99mTc]-TRODAT-1 SPECT study. Mov Disord. 2007; 22: 863-6.

Shulman LM, Taback RL, Bean J, Weiner WJ. Comorbity of the nonmotor symptoms of Parkinson's disease. Mov Disord. 2001;16: 507-10. 
Siderowf A, Newberg A, Chou KL, Lloyd M, Colcher A, Hurtig HI, Stern MB, Doty RL, Mozley PD, Wintering N, Duda JE, Weintraub D, Moberg PJ. [99mTc]TRODAT-1 SPECT imaging correlates with odor identification in early Parkinson disease. Neurology. 2005; 64:1716-20.

Silveira-Moriyama L, Carvalho Mde J, Katzenschlager R, Petrie A, Ranvaud R, Barbosa ER, Lees AJ. The Use of Smell Identification Tests in the Diagnosis of Parkinson's Disease in Brazil. Mov Disord. 2008; 23: 2328-34.

Štenc Bradvica I, Mihaljević I, Butković-Soldo S, Kadojić D, Titlić M, Bradvica M, Kralik K. Transcranial sonography and the pocket smell test in the differential diagnosis between parkinson's disease and essential tremor. Neurol Sci. 2015; 36: 1403-10.

Tissingh G, Bergmans P, Booij J, Winogrodzka A, Stoof JC, Wolters EC, Van Royen EA. [123I]beta-CIT single-photon emission tomography in Parkinson's disease reveals a smaller decline in dopamine transporters with age than in controls. Eur J Nucl Med. 1997; 24: 1171-4.

Tunc S, Graf J, Tadic V, Brüggemann N, Schmidt A, Al-Khaled M, Wolff S, Vollstedt EJ, Lorwin A, Hampf J, Piskol L, Klein C, Hagenah J, Kasten M. A Population-Based Study on Combined Markers for Early Parkinson's Disease. Mov Disord. 2015; 30: 531-7. 
Varrone A, Marek KL, Jennings D, Innis RB, Seibyl JP. [123I]ß-CIT SPECT imaging demonstrates reduced density of striatal dopamine transporters in Parkinson's disease and multiple system atrophy. Mov Disord. 2001; 16: 1023-32.

Walter U, Niehaus L, Probst T, Benecke R, Meyer BU, Dressler D. Brain parenchyma sonography discriminates Parkinson's disease and atypical parkinsonian syndromes. Neurology. 2003; 60: 74-7.

Walter U, Dressler D, Wolters A, Probst T, Grossmann A, Benecke R. Sonographic discrimination of corticobasal degeneration vs progressive supranuclear palsy. Neurology. 2004; 63: 504-9.

Walter U, Behnke S, Eyding J, Niehaus L, Postert T, Seidel G, Berg D. Transcranial brain parenchyma sonography in movement disorders: state of the art. Ultrasound Med Biol. 2007a; 33: 15-25.

Walter U, Dressler D, Probst T, Wolters A, Abu-Mugheisib M, Wittstock M, Benecke R. Transcranial Brain Sonography Findings in Discriminating Between Parkinsonism and Idiopathic Parkinson Disease. Arch Neurol. 2007b; 64:1635-40.

Walter U, Heilmann R, Kaulitz L, Just T, Krause BJ, Benecke R, Höppner J. Prediction of Parkinson's disease subsequent to severe depression: a ten-year follow-up study. J Neural Transm (Vienna). 2015; 122: 789-97. 
Wang J, Jiang YP, Liu XD, Chen ZP, Yang LQ, Liu CJ, Xiang JD, Su HL. 99mTcTRODAT-1 SPECT study in early Parkinson's disease and essential tremor. Acta Neurol Scand. 2005; 112: 380-5.

Wenning GK, Shephard B, Hawkes C, Petruckevitch A, Lees A, Quinn N. Olfactory function in atypical parkinsonian syndromes. Acta Neurol Scand. 1995; 91: 247-50.

Wirdefeldt K, Adami HO, Cole P, Trichopoulos D, Mandel J. Epidemiology and etiology of Parkinson's disease: a review of the evidence. Eur J Epidemiol. 2011; 26: S1-58.

Wu H, Lou C, Huang Z, Shi G. SPECT imaging of dopamine transporters with (99m)Tc-TRODAT-1 in major depression and Parkinson's disease. J Neuropsychiatry Clin Neurosci. 2011; 23: 63-7.

Xiao Q, Chen S, Le W. Hyposmia: a possible biomarker of Parkinson's disease. Neurosci Bull. 2014; 30: 134-40. 
APÊNDICES 
Artigo A: Revisão sistemática sobre importância da USTC

\section{Prospective studies on the role of transcranial sonography in defining Substantia Nigra Hyperechogenicity as a biomarker for idiopathic Parkinson's disease: A systematic review.}

Laboratory of Neurossonology, and neurocritical care. Clínicas Hospital. Faculty of Medicine, University of São Paulo.

\section{Corresponding Author:}

Kelson James Silva de Almeida

Transcranial doppler laboratory - Neurossonology group.

Central Institute. Eneas de Carvalho Aguiar Avenue, number 255, 5 floor.

Postal code: 05403-000. São Paulo - SP - Brazil.

Key Words: Parkinson disease; Substantia nigra; Ultrasonography; Smell; Olfaction disorders; Transcranial sonography. 


\section{Abstract}

Background:

Transcranial sonography (TCS) of the substantia nigra (SN) is increasingly used as a biomarker for idiopathic Parkinson's disease (PD). Few longitudinal studies investigating $\mathrm{SN}$ hyperechogenicity $(\mathrm{SNH})$ as a risk factor for the development of PD have been published.

Method:

A systematic review was performed on studies published as late as September 2015 that conducted TCS at baseline and at a follow-up visit to evaluate patients for PD.

Results:

Seven studies (ten published articles) used a prospective methodology and followed patients who received TCS at baseline to determine whether they were later diagnosed with idiopathic PD. However, these populations comprised healthy patients or individuals who presented various PD pre-motor markers.

Conclusions:

As few studies have been published, there is a lack of evidence supporting the high value of TCS for accurate PD diagnosis. SNH may be considered a reliable marker of nigrostriatal impairment and a strong risk factor for PD development based on the results of ongoing studies. 


\section{Introduction}

Parkinson's disease (PD) is the most prevalent neurodegenerative disease after Alzheimer's disease (AD) (1). However, the diagnosis of PD is based solely on clinical criteria, and there is no accurate biological or imaging marker to verify these clinical findings (2). Unfortunately, a substantial fraction of patients with PD are misdiagnosed, especially in the early stages of the disease (1). Another problem is the difficulty in differentiating tremor-dominant PD from essential tremor or from subjects without evidence of dopaminergic deficits (SWEDDs) (3).

There is increasing evidence from epidemiological surveys and clinical studies that non-motor symptoms may occur early and before motor symptoms, which should allow the diagnosis of a pre-motor PD state. Among these putative premotor markers of PD, dysautonomia, olfactory deficits, REM sleep behavior disorder, depression, and mild cognitive deficits are the most intensively investigated signs. To make it possible to develop an earlier, disease-modulating or even neuroprotective therapy, it is important to identify the subjects in a pre-motor PD state, when a significant portion of the neurons of the substantia nigra (SN) remain functional (4). SN hyperechogenicity (SNH) has been used as a biomarker for PD, as well as a risk variable associated with other non-motor symptoms, to predict the occurrence of PD. Based on these factors, this study was performed to review longitudinal studies that used TCS as a potential biomarker for PD. 
Technical Aspects

TCS should be performed according to the following steps. The examination should be conducted through the left and right temporal acoustic bone windows with a penetration depth of $16 \mathrm{~cm}$, a dynamic range of $50 \mathrm{~dB}$ and a mechanical index of 1.6. The image brightness should be adapted when necessary using B-mode gain and lateral gain control. The mesencephalic brainstem should be identified by its butterfly shape. After capturing the image, the structure image should be enlarged twofold. Within this structure, the hyperechogenic signal of the ipsilateral SN should be identified. A structure is classified as hyperechogenic if the intensity of the ultrasound signal is abnormally high compared with the reference structure, which is usually the surrounding white matter. The measurement should be taken after optimization of the signal at its largest extension. The area of the echogenic SN should be manually outlined using the cursor. The area is then calculated automatically. The median Raphe and ipsilateral red nuclei should be identified. Within the diencephalic insonation plane, the contralateral thalamus should be visible, and the width of the third ventricle and the contralateral frontal horn of the lateral ventricle measured perpendicular at the maximal width. The contralateral thalamic, caudate and lentiform nuclei should also be identified. This methodology was described according to the international consensus for TCS examination (5). 


\section{Methods}

A systematic search was performed in the Medline, EMBASE and

Scielo/Lilacs databases for articles published between January 1987 and September 2015 using the search terms "transcranial sonography", "substantia nigra hyperechogenicity”, "idiopathic REM sleep behavior disorder”, "hyposmia”, "depression", and "Parkinson disease" to identify publications that contained a prospective design and reported the use of TCS as a potential biomarker for PD. In these studies, the development of PD as an outcome must be consistent with the current diagnostic criteria.

The search strategies included the use of "transcranial sonography" OR "substantia nigra hyperechogenicity" AND a premotor marker (idiopathic REM sleep behavior disorder OR hyposmia OE depression) OR Parkinson disease. All possible combinations were searched.

Two investigators (KJA, EB) screened the full texts of the potentially relevant articles. Papers were included in the review if the following methodological information was given. 1) Studies described a TCS examination consistent with the international consensus (27) for ultrasound examination or after clarifying the neurosonologists' experiences. 2) A description of each center score for the median of the $75^{\text {th }}$ percentile of the right and left SN side according to defined criteria for SNH. 3) The outcome was the development of PD according to the current diagnostic criteria (the British Brain Bank criteria).

Papers were excluded if the following conditions were met: 1) the full article was not available, 2) the language was not English and 3) the study did not include patients without parkinsonism. Quality assessment of the 
diagnostic accuracy of the studies was performed using the QUADAS tool (table 01). The American Academy of Neurology (AAN) rating system (6) was used to classify the evidence in the selected studies.

\section{Results}

Overview

Seven studies (ten published articles) met the inclusion criteria. We did not find any additional clinical studies in the Cochrane databases. A cross-reference search added no other relevant publications. Two prospective studies were excluded because of a lack of TCS analysis at baseline (Honolulu and PARS studies). Another two studies were excluded because the published articles only described an ongoing recruitment methodology (TRENDS and PMPP studies).

We found a large population-based cohort study in which the results were reported in two published articles. The first article was published after monitoring the subjects for 37 months (7), and the second study was published after a 5-year follow-up period (8). Another cohort study was selected because its sample included patients with hyposmia who were subjected to TCS at baseline (9). A third cohort was added because TCS was performed at baseline on its sample group with rapid-eye movement sleep behavior disorder (RBD) (10). Although the primary outcome was neurodegenerative disease development in this last study, a subgroup analysis with PD diagnosis as the outcome was possible. A fourth study was included because it followed 196 
patients with unclear parkinsonism and TCS and SPECT were performed at baseline (11). The relative risk for a PD diagnosis was expressed for each population in the selected studies.

\section{Discussion}

In this review, we included 7 studies involving the use of TCS (presence of $\mathrm{SNH}$ ) as a risk factor for PD development. The results are discussed separately for the following: A) neuropathology in Parkinson's disease, B) TCS in Parkinson's disease, C) unselected cohort studies that aimed to diagnose PD, and E) selected prospective studies with TCS.

Neuropathology in Parkinson's Disease

The neuropathological features of Parkinson's disease (PD) include cell loss and a-synuclein aggregation (Lewy bodies and Lewy neurites) in multiple brain areas, including the $\mathrm{SN}$ of the midbrain. In addition, microglial activation and iron accumulation are also found (7). Studies have demonstrated that at the time of PD clinical diagnosis, a $40 \%$ to $60 \%$ loss of dopaminergic neurons has already occurred during a prediagnostic period estimated to be 5 to 15 years long $(12)$.

This concept of a pre-dopaminergic lesion in PD is supported by Braak's hypothesis stating that Lewy bodies and Lewy neurites develop in PD prior to the appearance of a nigrostriatal dopaminergic lesion at Braak stage 3. This PD-related synuclein pathology spreads in a caudal/rostral manner with an 
early involvement of the anterior olfactory nucleus and the lower brainstem (dorsal motor nucleus of the vagus), resulting in olfactory dysfunction, autonomic disturbances, depression, and rapid eye movement sleep behavior disorder (13).

With the establishment of SNH as a PD biomarker, a pathological link between the causes of SNH and the physiopathology of PD has been studied. The echogenicity of the SN in 3- to 4-month-old Wistar rats unilaterally injected with various concentrations of iron, ferritin, 6-hydroxydopamin, a combination of 6-hydroxydopamin and desferrioxamine, or zinc were compared with that of the non-injected contralateral side after 1 week. The comparisons revealed a dose-dependent increase in the area of SN echogenicity following the injection of iron or 6-hydroxydopamin. This same effect of iron on tissue echogenicity was found in a post-mortem study. An ultrasound examination of 60 brains immediately after autopsy and the relation between the area of hyperechogenicity and the results of histological and biochemical investigations revealed that there is a significant association between an increasing area of SN echogenicity and an increasing iron level. Additionally, in the SN of individuals with and without PD, an association between iron and an increase in microglia activation was detected (14). Berg et al confirmed that microglial activation was associated with the presence of $\mathrm{SNH}$ after examining sixty postmortem brains (15). These studies demonstrate that $\mathrm{SNH}$ can be associated with neuropathological features common in PD, such as increased SN iron content, decreased neuromelanin content, and SN microglial activation. 
TCS in Parkinson's Disease

Transcranial B-mode ultrasonography (TCS) is a noninvasive imaging technique that was initially developed to evaluate cerebrovascular disorders, particularly arterial stenosis and occlusion. Since the mid 1990s, however, TCS has evolved to enable the visualization of brain parenchymal structures and has been more recently used for the differential diagnosis of PD and other movement disorders. Becker et al first observed hyperechogenicity of the SN in PD using TCS $(9,34)$. Transcranial sonography (TCS) revealed that $90 \%$ of patients with PD and only approximately $10 \%$ of elderly individuals without PD had enlarged midbrain hyperechogenicity in the area of the SN. Additionally, many of these individuals demonstrated reduced fluorodopa uptake on PET, indicating the possibility that some normal individuals with SNH may already have a functional deficit in the nigrostriatal system and may be destined to develop PD (16).

Moreover, $\mathrm{SNH}$ is a stable marker because the area of echogenicity is not related to the disease stage and was found to remain unchanged as the disease progressed over a 5-year follow-up period (17). SNH appears to be a marker for the disease and not its progression (4). Furthermore, the prevalence of SNH in PD varies between $68-99 \%$ of cases. It has been shown that $\mathrm{SNH}$ is detectable at very early disease stages and that patients with bilateral rigidity and bilateral bradykinesia had significantly greater SN echogenicity and enlargement than did patients in whom these symptoms are unilaterally present (18). Berg et al showed that a hyperechoic and enlarged SN occurred more frequently on the side contralateral to the side with the more 
pronounced clinical symptoms (19). Moreover, the results from a 5-year followup study suggest that the size of the area with increased echogenicity does not change during the course of the disease (20).

Autosomal recessive and dominant genetic forms of PD are also associated with this increased echo signal. This association was found for the Parkin (PARK2) and PINK1 (PARK6), as well as the LRRK2 (PARK8) and glucocerebrosidase (GBA) genes (22). Brüggemann et al performed TCS in 34 LRRK2 G2019S mutation carriers manifesting PD, 24 non-manifesting mutation carriers, and 28 idiopathic PD patients and compared the results with those from 40 healthy controls. They proposed that the TCS results of patients with the common G2019S mutation in the LRRK2 gene were not substantially different from the results of the idiopathic PD patients.

Because TCS is noninvasive, economical, quick and easy to perform by properly trained examiners, it has the potential to be used as a secondary screening instrument in population-based screening batteries (7).

Unselected cohort studies aimed to diagnose PD

\section{TCS in PD patients with Depression}

Depression is not an uncommon symptom in patients with Parkinson's disease. Despite its wide incidence, affecting $4 \%$ to $70 \%$ of PD patients, approximately $40 \%$ of patients with PD experience a clinically significant depressive episode during the course of their illness. Depression has been suggested to be either an early symptom of PD or an associated risk 
factor for PD, considering that the risk of developing PD is known to be higher in depressed patients than in non-depressed controls. Choo et al. (3) studied 61 patients with PD (29 PD without depression, 32 PD with depression) and 41 controls. Their results indicated that decreased brainstem raphe echogenicity was much higher in PD patients with depression than in those without depression (3). Walter et al studied 55 controls without depression and without PD, 55 subjects with depression and without PD, 45 PD patients without depression and 45 PD patients with depression. They found that reduced echogenicity of the brainstem raphe was more frequent in the depressed subjects than in the non-depressed subjects, regardless of the PD diagnosis. The combination of both a marked $\mathrm{SNH}$ and a reduced raphe echogenicity in Parkinson's disease patients, however, was clearly associated with a history of depression prior to the onset of Parkinson's disease (20).

The value of TCS in the differential diagnosis of PD versus depression is most obvious during the earliest stages of the disease, when clinical features alone do not often allow for the diagnosis of either PD or depression in a patient. However, even in the later stages of PD, when hypomimia and bradykinesia may disguise a depressed mood, the easily obtainable TCS results may be very helpful. Moreover, the results of a recent study suggest that depressed patients (without PD) with raphe hypoechogenicity respond better to therapy with serotonin reuptake inhibitors than depressed subjects who do not present this echo feature. Future studies need to be conducted to determine whether TCS can be used to guide decisions regarding therapeutic treatment (21). A prospective follow-up study on 
a cohort of patients with decreased brainstem raphe echogenicity and SNH is needed to determine if these individuals will develop clinically defined PD.

\section{Cohort studies with other prodromal markers of PD}

As our understanding increases of the symptoms that may indicate future PD onset, the discussion on how to define the risk population for PD has gained increasing attention. Studies of cohorts at risk for PD can provide insight into the progression of the preclinical phase of the disease and can also be used to recruit subjects willing to participate in clinical trials for potential disease-modifying therapies (12).

In a Honolulu-Asia Aging Study cohort (HAAS), 2,267 men aged 71 to 95 years who were free of clinical PD and dementia were followed after olfactory testing using the Brief Smell Identification Test. The age-adjusted odds for the incidence of PD were greater than 5 for those in the lowest scoring quartile relative to those in the higher quartiles of the smell test. This study demonstrated that odor identification deficits could predate the development of clinical PD in men by at least 4 years (22). Interestingly, there was no relation between olfaction and incidence of PD during the second 4-year period, suggesting that olfactory deficits begin to occur within 4 years prior to the development of classic motor features that lead to a diagnosis (12).

The PARS study (Parkinson At-Risk Syndrome Study) used a twostage screening strategy for PD that consisted of olfactory testing followed by DAT imaging. This study described a range of PD prodromal features, including hyposmia, RBD symptoms, anxiety and depression, constipation, and self- 
reported change in motor function in approximately 5,000 individual with no diagnosis of PD. The association between hyposmia and these features was modest but detectable, considering the size of the PARS cohort. In phase II of the PARS study, 203 hyposmic subjects and a control group of 100 normosmic subjects underwent dopamine-transporter imaging and several additional assessments. A current model of progression has been tested to predict the time at which premotor PD subjects are likely to convert to motor PD.

The HAAS cohort confirmed that constipation is also associated with the future development of PD. The age-adjusted incidence of PD per 10,000 individuals in years presented a negative correlation with the number of bowel movements. The risk was highest among men who reported using laxatives (23). In the Honolulu-Asia Aging Study and the PARS study, TCS was not performed at baseline to define the TCS-based risk for PD.

\section{Ongoing unpublished longitudinal studies with TCS}

These unpublished longitudinal studies suggested that each single marker alone was not found in individuals at risk for the future onset of PD. Instead, combinations of risk markers increased the specificity and predictive positive value for PD development compared with those of single factors. Little is known about the prevalence, co-occurrence and progression of prodromal markers before occurrence of the clinical symptoms that enable PD diagnosis. The PMPP (Progression Markers in the Pre-motor Phase) study is an ongoing study designed to investigate the progression of various biomarkers during the prodromal phase of PD. This is the first study aiming to comprehensively 
assess individuals at high risk for idiopathic PD at short (6-month) intervals (1). In this study, individuals with SNH in addition to at least one PD motor sign or two other premotor/slight motor symptoms or a combination of these pre-early motor symptoms and a positive family history of PD are evaluated.

The TRENDS study selected individuals older than 50 years of age who presented idiopathic hyposmia and/or depression and/or self-reported signs of RBD using the RBD screening questionnaire. Currently, the recruited cohort includes almost 1,200 individuals. The investigations include assessments of motor performance, olfactory function, vision autonomic function, and cognition, as well as an ultrasound of the brain parenchyma and a measurement of the intima media thickness (IMT) of the carotids (12). This ongoing study has only published its protocol and was therefore excluded from this review. However, it should be mentioned because it employs a welldesigned enrollment strategy.

Selected prospective studies with TCS

\section{TCS in parkinsonism syndrome}

A prospective investigation of 60 patients with mild signs of unclear parkinsonism who were subjected to a TCS evaluation at baseline and then followed for 12 months was selected for the present review based on the methodology described above. After the follow-up period, 43 patients were diagnosed with idiopathic PD and 13 patients were diagnosed with atypical parkinsonism. Parkinsonism was ruled out in four patients. TCS was able to 
predict this diagnosis at baseline with high diagnostic accuracy. The sensitivity of $\mathrm{SNH}$ for iPD was $90.7 \%$, the specificity was $82.4 \%$, the positive predictive value was $92.9 \%$, and the negative predictive value was $77.8 \%$. The classification accuracy of $\mathrm{SN}$ echogenicity at baseline compared with the endpoint diagnosis was $88.3 \%$ (24). The evidence level for this study was Class II.

Another study followed 196 patients with unclear parkinsonism for 2 years. The authors concluded that the diagnostic accuracy of TCS during the early stage of parkinsonism was not sufficient for routine clinical use. In this study, only $52 \%$ of patients had a final diagnosis of IPD. For the final diagnosis, other conditions were found, including vascular parkinsonism (in 10.2\% of patients), drug-induced parkinsonism (in 3.6\% of patients) and no parkinsonism but rather an alternative diagnosis (in $11.2 \%$ of patients). The sensitivity of SNH for IPD diagnosis was $40 \%(\mathrm{Cl} 0.30$ to 0.50$)$, and the specificity was $61 \%(\mathrm{Cl}$ 0.52 to 0.70 ), supporting the authors' conclusion (11). The rating of the evidence level was Class II for this study. However, it should be noted that certain conditions, such as vascular parkinsonism, could be excluded at baseline using this methodology. Magnetic resonance images were only considered during the final diagnostic analysis at the end of the two-year follow-up period. An inclusion bias in $10.2 \%$ of the patients may account for these results.

\section{$\underline{\text { TCS in patients with rapid-eye-movement sleep behavior disorder }}$}

Clinical aspects of the premotor phase of PD include constipation, daytime sleepiness, RBD, affective symptoms, and impaired sense of smell 
(25). RBD is characterized by dream-enacting behaviors that are linked to REM sleep without atonia. Idiopathic RBD (iRBD) typically develops in middle-aged individuals or older individuals and progresses chronically. RBD is a common clinical manifestation of Lewy body-related syndrome $(10,16)$. Iranzo et al followed 43 individuals with $\mathrm{IRBD}$ and 149 controls for 2-5 years after neuroimaging procedures. Four patients with iRBD had an insufficient bone window. SNH was found in $14(36 \%)$ of 39 participants with iRBD compared with $16(11 \%)$ of 149 controls $(p \leq 0.002)$. The mean echogenicity of the SN was larger in the iRBD patients than in the controls. None of the 15 participants with normal 123I-FP-CIT binding (SPECT) and echogenicity developed a neurological syndrome during the 29-month follow-up period. Five patients with SNH and RBD at baseline developed PD during the follow-up period. In this group, the relative risk for PD development was 2.67 . The evidence level for these results was Class II according to the American Academy of Neurology rating system.

This same research group published another study that included 55 iRBD patients who underwent TCS at baseline. Nineteen (34.5\%) patients had positive SNH, $32(58.2 \%)$ demonstrated negative SN, and $4(7.3 \%)$ had an insufficient temporal acoustic bone window. Five years after the baseline TCS, among the 19 patients who were SN+ at baseline, $10(52.7 \%)$ remained disease-free, eight (42.0\%) developed a synucleinopathy (four PD and four Dementia of Lewy Body), and one (5.3\%) was lost to follow-up. Of the 32 patients who were SN- at baseline, 21 (65.6\%) remained disease-free and 11 (34.4\%) developed a synucleinopathy (five PD, five Dementia of Lewy Body, and one Multiple System Atrophy) (26). 


\section{TCS in patients with hyposmia}

A small sample study followed 24 patients with idiopathic olfactory loss to determine the percentage of patients who developed IPD after a 4-year period. A total of $7 \%$ of the individuals with idiopathic hyposmia developed clinical IPD, and $13 \%$ of the patients presented relevant abnormalities of the motor system. At baseline, 11 patients presented SNH during the TCS evaluation. In this subgroup, 3 patients developed motor abnormalities. After the 4-year period, none of the 14 patients with normal findings from the TCS developed clinically relevant motor abnormalities (9). Upon analysis with the QUADAS tool, it was found that the international consensus (5) for ultrasound examination was not cited in the methodology. The number of patients who were subjected to TCS and had an insufficient bone window, which suggests that the results might be uninterpretable, was unclear. The evidence level rating of the diagnostic/prognostic TCS value in this study was Class II.

\section{TCS in patients with depression}

Walter et al followed 57 patients with major depressive disorder (MDD) over a median time of 10 years. The baseline evaluation included transcranial sonography and tests of olfactory function, depression severity, and dementia. A total of 17 patients were lost during the follow-up period. Three $(7.5 \%)$ of the follow-up patients were diagnosed with definite PD. All of the patients who developed clinically definite PD tested positive for idiopathic hyposmia and were $\mathrm{SN}+$. The authors suggested that MDD patients at risk for 
subsequent PD can be identified using an inexpensive non-invasive diagnostic battery that assesses tobacco use, constipation, olfaction, movement of the upper face and extremities, and SN echogenicity (27).

\section{Long-term studies with TCS}

The PRIPS study (Prospective Validation of Risk Factors for the Development of Parkinsonian Syndromes) was a multicenter prospective cohort study designed to define the value of midbrain ultrasonography for detecting preclinical PD. In this study, the relative risk for the incidence of PD was more than 17 times higher in the elderly participants with SNH compared with those without $\mathrm{SNH}$, thus demonstrating an association between $\mathrm{SNH}$ and the subsequent development of PD in healthy adults during the 37-month follow-up period. Other preclinical PD markers, such as depression, hyposmia and RBD, were not considered to be more relevant than the TCS results in this study (7). According to the QUADAS evaluation, the patient's withdrawals were not explained in the results.

The second follow-up examination of the PRIPS study was performed on 1,271 subjects 5 years after the baseline examination. After 5 years, 21 individuals were diagnosed with PD, 11 of whom had already been diagnosed with PD at a 3-year follow-up examination. Among these 21 individuals diagnosed with PD, the echogenicity of the SN could be evaluated in 17 , and $14(77.8 \%)$ displayed $\mathrm{SNH}$ at the baseline TCS assessment. The magnitude of the increase in echogenicity does not appear to change during the course of the disease. The 
SNH status was associated with an increased relative risk for the subsequent development of PD in healthy adults. This cohort study showed that this echo feature, when present in healthy subjects 50 years or older, is associated with approximately the highest relative risk of any risk and prodromal marker currently reported for $\operatorname{PD}(8,28,29)$. However, 565 individuals were lost over the course of the study (participation rate: $69.4 \%$ ). This result was again not explained. The PRIPS study, however, has a Class I evidence level rating because it followed a broad spectrum of individuals with a suspected condition.

\section{Conclusion}

Longitudinal studies with TCS would improve PD diagnosis at very early stages and allow drugs with potential disease-modifying effects to be tested before motor and cognitive symptoms emerge (10). Furthermore, closely monitoring individuals with preclinical disease would allow the early diagnosis of symptom onset and treatment, which may improve their health status.

This current review emphasizes the role of TCS in idiopathic PD diagnosis based on prospective methodologies. Because few studies included baseline TCS in their prospective designs, we propose that there is a lack of evidence supporting the high value of TCS for accurate PD diagnosis.

However, SNH may be considered a marker of nigrostriatal impairment and a strong risk factor for the development of iPD. It is more probable that the role of SNH may vary across each sample subgroup. More prospective studies are needed, especially to define a more reliable subgroup in which SNH identification could be valuable for routine clinical use and for guiding the assessment of disease-modifying therapies. 
Table 01: Analysis of selected studies using the QUADAS tool. Legend: 1-

"Yes", 2- "No", 3- "Unclear"

\begin{tabular}{|c|c|c|c|c|c|c|c|}
\hline Item/Reference & $\begin{array}{l}\text { Berg } D . \\
\text { et al }(7 \\
8)\end{array}$ & $\begin{array}{l}\mathrm{Haeh} \\
\text { ner et } \\
\text { al (9) }\end{array}$ & $\begin{array}{l}\text { Iran } \\
\text { zo et } \\
\text { a I } \\
(10)\end{array}$ & $\begin{array}{l}\text { Gaen } \\
\text { s le n } \\
\text { et al } \\
(24)\end{array}$ & $\begin{array}{l}\text { Bouw } \\
\text { mans } \\
\text { et al, } \\
(11)\end{array}$ & $\begin{array}{l}\text { Iranzo } \\
\text { et al } \\
(26)\end{array}$ & $\begin{array}{l}\text { Walter U } \\
\text { et al } \\
(27)\end{array}$ \\
\hline $\begin{array}{l}\text { 1. Was the spectrum of patients } \\
\text { representative of the patients who } \\
\text { will receive the test in practice? }\end{array}$ & 1 & 1 & 1 & 1 & 2 & 1 & 1 \\
\hline $\begin{array}{l}\text { 2. Were the selection criteria clearly } \\
\text { described? }\end{array}$ & 1 & 1 & 1 & 1 & 1 & 1 & 1 \\
\hline $\begin{array}{l}\text { 3. Is the reference standard likely to } \\
\text { correctly classify the target } \\
\text { condition? }\end{array}$ & 1 & 1 & 1 & 1 & 1 & 1 & 1 \\
\hline $\begin{array}{l}\text { 4. Is the time period between the } \\
\text { reference standard and index test } \\
\text { short enough to be reasonably sure } \\
\text { that the target condition did not } \\
\text { change between the two tests? }\end{array}$ & 1 & 1 & 1 & 1 & 1 & 1 & 1 \\
\hline $\begin{array}{l}\text { 5. Was the whole sample or a } \\
\text { random selection of the sample } \\
\text { verified using a reference standard of } \\
\text { diagnosis? }\end{array}$ & 1 & 1 & 1 & 1 & 1 & 1 & 1 \\
\hline $\begin{array}{l}\text { 6. Did patients receive the same } \\
\text { reference standard regardless of the } \\
\text { index test result? }\end{array}$ & 1 & 1 & 1 & 1 & 1 & 1 & 1 \\
\hline $\begin{array}{l}\text { 7. Was the reference standard } \\
\text { independent of the index test (i.e., } \\
\text { the index test did not form part of the } \\
\text { reference standard)? }\end{array}$ & 1 & 1 & 1 & 1 & 1 & 1 & 1 \\
\hline $\begin{array}{l}\text { 8. Was the execution of the index } \\
\text { test described in sufficient detail to } \\
\text { permit replication of the test? }\end{array}$ & 1 & 2 & 1 & 1 & 1 & 1 & 1 \\
\hline $\begin{array}{l}\text { 9. Was the execution of the reference } \\
\text { standard described in sufficient detail } \\
\text { to permit its replication? }\end{array}$ & 1 & 1 & 1 & 1 & 1 & 1 & 1 \\
\hline $\begin{array}{l}\text { 10. Were the index test results } \\
\text { interpreted without knowledge of the } \\
\text { reference standard results? }\end{array}$ & 1 & 1 & 1 & 1 & 1 & 1 & 1 \\
\hline $\begin{array}{l}\text { 11. Were the reference standard } \\
\text { results interpreted without knowledge } \\
\text { of the index test results? }\end{array}$ & 1 & 1 & 1 & 1 & 1 & 1 & 1 \\
\hline $\begin{array}{l}\text { 12. Were the same clinical data } \\
\text { available when the test results were } \\
\text { interpreted as would be available } \\
\text { when the test is used in practice? }\end{array}$ & 1 & 1 & 1 & 1 & 1 & 1 & 1 \\
\hline $\begin{array}{l}\text { 13. Were uninterpretable/ } \\
\text { intermediate test results reported? }\end{array}$ & 1 & 3 & 1 & 1 & 1 & 1 & 1 \\
\hline $\begin{array}{l}\text { 14. Were withdrawals from the study } \\
\text { explained? }\end{array}$ & 2 & 1 & 1 & 1 & 1 & 1 & 1 \\
\hline
\end{tabular}




\section{References}

1. Liepelt-Scarfone I, Gauss K, Maetzer W, Muller K, Bormann C, Fruhmann Berger M, Timmers M, Streffer J, Berg D. Evaluation of progression phase in the Premotor Phase of Parkinson's Disease: The Progression Markers in the Premotor Phase Study. Neuroepidemiology. 2013; 41(3-4):174-182.

2. Laučkaitè K, Rastenytè D, Šurkienè D, Vaitkus A, Sakalauskas A, Lukoševičius A, Gleiznienè R. Specificity of transcranial sonography in parkinson spectrum disorders in comparison to degenerative cognitive syndromes. BMC Neurol. 2012; 12:12.

3. Cho JW, Baik JS, Lee MS. Mesencephalic midline change on transcranial sonography in early Parkinson's disease patients with depression. J Neurol Sci. 2011; 310(1-2):50-2.

4. Berg D. Substantia nigra hyperechogenicity is a risk marker of Parkinson's disease: yes. J Neural Transm. 2011; 118(4):613-9.

5. Walter U, Behnke S, Eyding J, Niehaus L, Postert T, Seidel G, Berg D. Transcranial brain parenchyma sonography in movement disorders: state of the art. Ultrasound Med Biol. 2007; 33(1):15-25. 
6. Goodin DS, Edlund W. Process for developing technology assessments. American Academy of Neurology Therapeutics and Technology Subcommittee, 1999: 1-35.

7. Berg D, Seppi K, Behnke S, Liepelt I, Schweitzer K, Stockner H, Wollenweber F, Gaenslen A, Mahlknecht P, Spiegel J, Godau J, Huber H, Srulijes K, Kiechl S, Bentele M, Gasperi A, Schubert T, Hiry T, Probst M, Schneider V, Klenk J, Sawires M, Willeit J, Maetzler W, Fassbender K, Gasser T, Poewe W. Enlarged substantia nigra hyperechogenicity and risk for Parkinson disease: a 37-month 3-center study of 1847 older persons. Arch Neurol. 2011; 68: 932-937.

8. Berg D, Behnke S, Seppi K, Godau J, Lerche S, Mahlknecht P, LiepeltScarfone I, Pausch C, Schneider N, Gaenslen A, Brockmann K, Srulijes K, Huber H, Wurster I, Stockner H, Kiechl S, Willeit J, Gasperi A, Fassbender K, Gasser T, Poewe W. Enlarged hyperechogenic substantia nigra as a risk marker for Parkinson's disease. Mov Disord. $2013 ; 28(2): 216-9$.

9. Haehner A, Hummel T, Hummel C, Sommer U, Junghanns S, Reichmann $\mathrm{H}$. Olfactory loss may be a first sign of idiopathic Parkinson's disease. Mov Disord. 2007; 22(6):839-42. 
10. Iranzo A, Lomeña F, Stockner H, Valldeoriola F, Vilaseca I, Salamero M. Decreased striatal dopamine transporter uptake and substantia nigra hyperechogenicity as risk markers of synucleinopathy in patients with idiopathic rapid-eye-movement sleep behaviour disorder: a prospective study. Lancet Neurol. 2010; 9: 1070-1077.

11. Bouwmans AEP, Vlaar AMM, Mess WH, et al. Specificity and sensitivity of transcranial sonography of the substantia nigra in the diagnosis of Parkinson's disease: prospective cohort study in 196 patients. BMJ. 2013; 3: 2613.

12. Berg D, Marek K, Ross GW, Poewe W. Defining at-risk populations for Parkinson's disease: lessons from ongoing studies. Mov Disord. 2012; 27: 656-665.

13. Godau J, Hussl A, Lolekha P, Stoessl AJ, Seppi K. Neuroimaging: Current Role in Detecting Pre-Motor Parkinson's Disease. Mov Disord. $2012 ; 27(5): 634-43$.

14. Groger A, Berg D. Does structural neuroimaging reveal a disturbance of iron metabolism in Parkinson's disease? Implications from MRI and TCS studies. J Neural Transm. 2012;119:1523-1528. 
15. Berg D, Godau J, Riederer P, Gerlach M, Arzberger T. Microglia activation is related to substantia nigra echogenicity. J Neural Transm. 2010 Nov;117(11):1287-92..

16. Miyamoto M, Miyamoto T. Neuroimaging of rapid eye movement sleep behavior disorder: transcranial ultrasound, single-photon emission computed tomography, and positron emission tomography scan data. Sleep Med. 2013; 14(8):739-43.

17. D. Berg, B. Merz, K. Reiners, M. Naumann, G. Becker. Five-year follow-up study of hyperechogenicity of the substantia nigra in Parkinson's disease. Mov Disord. 2005; 20: 383-385.

18. Bártová P, Skoloudík D, Ressner P, Langová K, Herzig R, Kanovsky P. Correlation between substantia nigra features detected by sonography and Parkinson disease symptoms. J Ultrasound Med. $2010 ; 29(1): 37-42$

19. Berg D, Siefker C, Becker G. Echogenicity of the substantia nigra in Parkinson's disease and its relation to clinical findings. J Neurol. 2001; 248: 684-689. 
20. Walter U, Hoeppner J, Prudente-Morrissey L, Horowski S, Herpertz SC, Benecke R. Parkinson's disease-like midbrain sonography abnormalities are frequent in depressive disorders. Brain. 2007; 130(Pt 7):1799-807.

21. Walter U, Skoloudík D, Berg D. Transcranial sonography findings related to non-motor features of Parkinson's disease. J Neurol Sci. $2010 ; 289(1-2): 123-7$.

22. Ross GW, Petrovitch H, Abbott RD, Tanner CM, Popper J, Masaki K, Launer L, White LR. Association of olfactory dysfunction with risk for future Parkinson's disease. Ann Neurol. 2008; 63: 167-173.

23. Abbott RD, Petrovitch $H$, White $L R$, et al. Frequency of bowel movements and the future risk of Parkinson's disease. Neurology. $2001 ; 57: 456-462$.

24. Gaenslen A, Unmuth B, Godau J, Liepelt I, Di Santo A, Schweitzer KJ, Gasser T, Machulla HJ, Reimold M, Marek K, Berg D. The specificity and sensitivity of transcranial ultrasound in the differential diagnosis of Parkinson's disease: a prospective blinded study. Lancet Neurol. $2008 ; 7(5): 417-24$. 
25. Siderowf A, Jennings D, Eberly S, Oakes D, Hawkins KA, Ascherio A, Stern MB, Marek K. Impaired olfaction and other prodromal features in the Parkinson At-Risk Syndrome Study. Mov Disord. 2012; 27: 406412.

26. Iranzo A, Stockner H, Serradell M, Seppi K, Valldeoriola F, Frauscher B, Molinuevo JL, Vilaseca I, Mitterling T, Gaig C, Vilas D, Santamaria J, Högl B, Tolosa E, Poewe W. Five-year follow-up of substantia nigra echogenicity in idiopathic REM sleep behavior disorder. Mov Disord. 2014; 29(14):1774-80.

27. Walter U, Heilmann R, Kaulitz L, Just T, Krause BJ, Benecke R, Höppner J. Prediction of Parkinson's disease subsequent to severe depression: a ten-year follow-up study. J Neural Transm. 2015; 122 (6): 789-797.

28. Berg D, Godau J, Seppi K, Behnke S, Liepelt-Scarfone I, Lerche S, Stockner H, Gaenslen A, Mahlknecht P, Huber H, Srulijes K, Klenk J, Fassbender K, Maetzler W, Poewe W; PRIPS study group. The PRIPS study: screening battery for subjects at risk for Parkinson's disease. Eur J Neurol. 2013; 20(1):102-8. 
29. Lerche S, Seppi K, Behnke S, Liepelt-Scarfone I, Godau J, Mahlknecht P, Gaenslen A, Brockmann K, Srulijes K, Huber H, Wurster I, Stockner H, Kiechl S, Willeit J, Gasperi A, Fassbender K, Poewe W, Berg D. Risk factors and prodromal markers and the development of Parkinson's disease. J Neurol. 2014; 261(1):180-7. 
Artigo B: Estudo sobre acurácia dos métodos combinados (USTC e SST-16) em comparação a acurácia do TRODAT para diagnóstico de Doença de Parkinson.

Combined assessment by transcranial sonography and Sniffin' Sticks test has a similar diagnostic accuracy compared to brain SPECT for Parkinson's disease diagnosis.

Laboratory of Neurossonology, and neurocritical care. Clínicas Hospital. Faculty of Medicine, University of São Paulo.

\section{Corresponding Author:}

Kelson James Silva de Almeida

Transcranial doppler laboratory - Neurossonology group.

Central Institute. Eneas de Carvalho Aguiar Avenue, number 255, 5 floor.

Postal code: 05403-000. São Paulo - SP - Brazil.

Key Words: Parkinson disease; Substantia nigra; Ultrasonography; Smell; Tomography, Emission-Computed, Single-Photon; Olfaction disorders. 


\section{Introduction}

Diagnosing Parkinson's disease (PD) can be challenging, especially in the early stages of the disease. More clinical trials investigating drugs with potential disease-modifying effects are needed for the early stages of the disease. In this picture, an accurate diagnosis requires more than clinical findings alone, and brain perfusion single-photon emission computed tomography (SPECT), Positron emission tomography (PET), or transcranial sonography (TCS) may be helpful in diagnosing PD and differentiating it from atypical parkinsonian syndromes and essential tremor (1).

Transcranial sonography (TCS) shows an increased echogenic size of the substantia nigra, known as substantia nigra hyperechogenicity (SNH), in $90 \%$ of patients with a PD diagnosis (2). In long-term studies, elderly patients with SNH at baseline determined via TCS had an increased relative risk of PD and were more than 17 times more likely to be diagnosed with PD than those without SNH (3). Such studies are part of the increasing epidemiological evidence and clinical studies that illustrate that $\mathrm{SNH}$ and other non-motor symptoms may be observed before motor symptoms or PD diagnosis.

Dysautonomia, hyposmia, REM sleep behavior disorder and depression are associated with PD, and soon SNH may be considered a PD biomarker $(3,4)$. Hyposmia is a disorder characterized by a decreased ability of detecting odors. PD patients more often have hyposmia compared to healthy counterparts (5). The Sniffin' Sticks test is an easily available test for a quick evaluation of hyposmia. Other tests for hyposmia are the Smell Identification 
Test and the Pocket Smell Test (5). However, few studies have used TCS in addition to other non-motor symptoms to perform PD diagnosis.

Dopamine transporter scan (DATSPECT) imaging is used to visualize the nigrostriatal dopamine pathway and molecular dopamine transporters. The density of the striatal dopamine transporters (DAT) is decreased in patients with PD. The striatal DAT density is a surrogate marker for the degeneration of dopaminergic neurons in the substantia nigra (SN). Recent studies have proven the high specificity (98.6\%) and sensitivity $(99.4 \%)$ of DATSPECT in confirming neurodegenerative parkinsonism (5). However, DATSPECT is an expensive and less widely available method. Considering the worldwide availability of the following methods to diagnose PD, this study aimed to investigate the accuracy of transcranial brain parenchyma sonography in addition to the Sniffin' Sticks Test (SST-16) for differentiation between idiopathic Parkinson's disease (PD) and controls compared to that of 99mTc-TRODAT-1 DATSPECT.

\section{Methods}

This was a prospective, cross-sectional study. Twenty patients with PD (13 males and 7 females), diagnosed in accordance with United Kingdom Parkinson's Disease Society Brain Bank criteria, were included in this study and received treatment from a Brazilian movement disorder outpatient clinic. Their ages ranged from 43 to 76 years and the median age was 62.5 years $(54 ; 67.5)$. Median age of onset of PD was 56.5 years $(49 ; 66.7)$, and the 
age at onset of disease ranged from 38-86 years, with a mean disease duration of one to fifteen years and a median of five years $(2.2 ; 5.7)$. One patient was graded as modified Hoehn and Yahr $(\mathrm{HY})$ stage one, nine patients as $\mathrm{HY}$ stage two, nine patients as HY stage three, and one patient as HY stage four. The median UPDRS-III score, assessed during the off phase, was 29 (17.5; 35.5). Nine age and sex-matched healthy subjects who worked at the hospital formed the control group. There were 6 males and 3 females in the control group. Their ages varied from 43 to 57 years, with a median of 51 years $(46 ; 54)$.

All patients were examined by a movement disorder specialist to exclude neurologic or clinical abnormalities in healthy subjects and to prove clinical criteria in PD patients. No family members of PD patients were included in the control group. All patients with a PD family history or with neuroleptic exposure were eliminated. PD patients and controls underwent brain SPECT using 99mTC-TRODAT-1 as the radiopharmaceutical and TCS examination. The time interval between the two examinations varied from six to 23 months. At time of TCS examination, clinical and epidemiological variables were collected, and the Sniffin' Sticks test for olfactory evaluation was performed. The clinically collected variables were the following: age, gender, age at initial symptoms, disease duration, and education time. The following scales were used to evaluate disease severity and the functional status of PD patients: Unified Parkinson's Disease Rating Scale (UPDRS) part III, modified Hoehn and Yahr (HY) Scale, and the Schwab and England Activities of Daily Living Scale (SE). We excluded smokers from the study if they had an active upper respiratory tract infection, chronic rhinitis or sinusitis, or if there was a previous history of 
psychiatric disease or head trauma leading to loss-of-consciousness. All patients and controls underwent a Mini Mental state examination (MMSE) using the validated Portuguese translation by Brucki et al (6). We excluded those whose score was less than cut-off points in accordance of years of schooling. Consent was obtained from all participants, and the protocol was approved by the local ethics committee.

\section{Brain SPECT images}

SPECT images were obtained $4 \mathrm{~h}$ after intravenous injection of $814 \mathrm{MBq}( \pm 74)$ 99mTc-TRODAT-1 (produced by the Institute of Nuclear Energy Research, Taiwan, Republic of China) using a dual-head camera coupled with fan beam collimators (Infinia ${ }^{\mathrm{TM}}$ Hawkeye ${ }^{\circledR}$ SPECT/CT, GE Healthcare). The TRODAT-1 kits were labeled and controlled before administration, and the radiochemical purity control was verified by the solvent extraction method. Images were reconstructed and reoriented based on the anteroposterior commissural line at a Xeleris workstation (GE Medical Systems). Photon attenuation correction was performed using Chang's first-order correction method 8. DAT density was calculated using the binding potential from the regions of interest $(\mathrm{ROI})$, bilaterally drawn in the striatum, caudate nucleus, putamen, and occipital lobe, by two independent readers. DAT binding potential was obtained according to the following equation: (specific binding nonspecific binding)/nonspecific binding. The lowest values of DAT binding potentials in the striatum were used from both sides of each participant for 
statistical analysis. Good inter-observer reproducibility was proven in a previously published paper about the same Brazilian PD patient cohort (7).

\section{The Sniffin' Sticks test}

The smell test was performed by means of the SST-16 (Burghart Medizintchnik, Gemany), which is commercially available, validated and translated into Portuguese $(8,9)$. The test consists of sixteen pens filled with common odorants: orange, leather, cinnamon, peppermint, banana, lemon, licorice, turpentine, garlic, coffee, apple, cloves, pineapple, rose, anise, and fish. The pens are approximately $14 \mathrm{~cm}$ in length and can be used for up to one year, according to manufacturer. During the test, the examiner removed the cap and placed the tip of the pen $2 \mathrm{~cm}$ from both nostrils of the patients. They were free to sample the odors as often as necessary until they were able to identify the odorant from a list of four options given by the examiner for each pen. If they were not able to identify it, they had to guess (forced multiple choice task identification test). The interval between each odorant was at least 30 seconds. SST-16 measures smell identification, threshold and discrimination. It was chosen identification measurement as variable to diagnosis olfactory impairment in this section. The test was performed in a quiet, well-ventilated room. 
Statistical analysis

The statistical analysis was performed using SPSS 20.0 Software. Median values and upper/lower quartiles (25th/75th percentiles) were used to describe demographic data. A normal distribution pattern was evaluated by the Shapiro-Wilk test. The Chi-square test was used for comparison between categorical variables. Parametric and non-parametric continuous variables were correlated by Student's $T$ test and the Mann-Whitney $U$ test, respectively. Spearman's rho coefficient was used for bivariate correlation between nonparametric variables. The Cohen's Kappa statistics were used for the evaluation of the compatibility between the methods. Receiver Operating Characteristic (ROC) curves were used to calculate cut-off points for TCS, Striatal DAT binding potentials and SST-16. The area under the ROC curve determined the accuracy of the PD diagnosis.

\section{Results}

In this study, there were more men than women in both groups, but there was no statistically significant difference in the gender and ages between the groups. The median age of the patients was $51(46 ; 54)$ in the PD group and $62.5(54 ; 67.5)$ in the control group. The median age at onset of the disease was 56.5 years $(49 ; 66.7)$. The disease duration of the PD group was $5.0(2.2 ; 5.7)$. The median disease severity scores were $29.0(17.5 ; 35.5)$ by UPDRS III and $2.2(2.0 ; 2.5)$ by modified HY stage. PD patients showed low 
scores on the SE scale (58.6\% with scores ranging from 80 to 100 points). The lower stages of PD severity were justified by the need to maintain blindness during TCS examination. Mean MMSE scores were $27.66 \pm 1.80$ in the control group and $26.00 \pm 3.59$ in the PD group, suggesting that cognitive impairments had no influence on the SST-16 results (Table 01).

\section{Transcranial sonography findings}

In the control group, the echogenic area of the $\mathrm{SN}$ had a mean of $0.27 \pm 0.23$, and the median was $0.17(0.14 ; 0.43)$. In the PD group, SN had as echogenic area with a mean of $0.51 \pm 0.14$ and a median of $0.51(0.40 ; 0.65)$. A larger SN echogenic area was observed in the PD group, with a statistically significant difference $(p=0.013)$ (Table 02). A cut-off point of $0.22 \mathrm{~cm}^{2}$ was obtained from a ROC curve for PD diagnosis. With this cut-off point, sensitivity was estimated at $100 \%$, with a specificity of $78 \%$ for the PD diagnosis. $100 \%$ of PD patients and $22.2 \%$ of healthy patients showed an SN echogenic area larger than the cut-off point $(p<0.001)$. An inadequate temporal acoustic bone window for midbrain disclosure was found on the right side in 1 PD patient and on the left side in another PD patient. These patients were excluded from the analysis.

\section{Brain 99mTc-TRODAT-1 SPECT}

ROI were bilaterally evaluated in the caudate, striatum and putamen. ROC curves were performed to determine the accuracy of the PD 
diagnosis after the ROI evaluation. The area under the curve and its 95\% intervals analyzed for the lowest and highest values of DAT binding potentials at caudate, striatum, putamen and were 0.975 (0.917-1.000), 0.998 (0.982-1.001), and $0.944(0.822-1.000)$, respectively. It was concluded that values of the striatum showed the best accuracy of PD diagnosis. The median striatal 99mTcTRODAT-1 SPECT binding values and their 25-75 percentiles are presented in table 02. The decrease in striatal 99mTc-TRODAT-1 SPECT binding was bilateral in all PD patients. Just one healthy volunteer had a value lower than the cut-off point in the striatal 99mTc-TRODAT-1 binding evaluation. This finding was more frequently observed in the PD group $(p<0.001)$. The cut-off value of 0.90 for striatal $99 \mathrm{mTC}-\mathrm{TRODAT}-1$ binding was associated with $100 \%$ sensitivity and an $89 \%$ specificity for the diagnosis of PD (area under the curve $=0.99 ; p<$ $0.001)$.

\section{Sniffin' Sticks test performance}

The control group scored the a median of 12 points $(11 ; 13)$ on the SST-16 test; the PD group scored a median of 9.00 points $(5.25 ; 10.75)$. This decrease in SST-16 performance observed in the PD group was of statistically significant $(p<0.001$, Table 02). The ROC curve results suggest a value equal or greater than 10 points at SST-16 to diagnose olfactory impairment in both groups (area under the curve $=0.858, p=0.02$ ). All healthy subjects scored normally on the SST-16. Olfactory dysfunction was observed in 13 (65.0\%) PD 
patients. Using this cut-off point, SST-16 showed a sensitivity of $65 \%$ and a specificity of $100 \%$ for PD diagnosis.

\section{Comparison between the methods for PD diagnosis}

There was no correlation observed between striatal 99mTcTRODAT-1 SPECT binding values and the echogenic area of the SN ( $r=$ 0.269, $p=0.159)$. A positive correlation between striatal 99mTc-TRODAT-1 SPECT binding values and SST-16 performance was observed to be statistically significant $(r=0.423, p=0.022)$. The SST-16 performance and echogenic area of SN via TCS had a negative correlation and Spearman's rho correlation was statistically significant $(r=-0.423, p=0.019)$.

Kappa statistics were used for the measurement of agreement between the tested methods and 99mTc-TRODAT-1 SPECT, considered to be the reference method during comparison. An excellent level of agreement was observed between 99mTc-TRODAT-1 SPECT and TCS, with a Kappa value of 0.92 (standard error= 0.77; p value < 0.001). A good agreement level was observed between SST-16 and the reference method of PD diagnosis, with Kappa value of 0.518 (standard error=0.132; p value $<0.001$ ).

In comparing the accuracy of the methods (table 04), striatal 99mTc-TRODAT-1 SPECT was the most accurate method tested. SST-16 alone had a better accuracy than TCS for PD diagnosis. When the two previously mentioned methods (SST-16 + TCS) were analyzed simultaneously, the accuracy approached that of striatal 99mTc-TRODAT-1 SPECT. These results suggest that a patient with abnormal changes in both tests (SST-16 + TCS) has 
Parkinson's Disease, with diagnosis likely to be confirmed by striatal 99mTcTRODAT-1 SPECT.

\section{Discussion}

PD diagnosis is based solely on clinical criteria. But in some cases, complementary tools are necessary in patients for whom there is a question of a PD diagnosis. This study provided useful clinical information after assess a new diagnostic test for PD (by combining two other tests). The diagnostic accuracy of two inexpensive and more widely available tests for diagnosing PD (TCS plus SST-16) was close to brain DATSPECT accuracy, which is expensive and less available method.

A community study in Brazil screened 1,186 subjects older than 64 years using a questionnaire and neurological examination and revealed that the prevalence of parkinsonism might be as high as $7.2 \% .72 \%$ of the 39 PD patients identified in this study had not been diagnosed by a primary care physician. It is estimated that 10 million Brazilians are older than 64 years old (10). Brazil has specialized centers that focus on dementia and movement disorders, but they are only located in the more populous cities. Currently, PD diagnosis is a clinical diagnosis, but in some cases, imaging methods are used to support the diagnosis. DATSPECT is both sensitive (92\%) and specific $(100 \%)$ in the evaluation of nigrostriatal dopamine deficiency in patients with suspected PD. However, in countries such as Brazil, where access to a specialized neurological center may be difficult, the use of DATSPECT to 
support PD diagnosis may be a challenge. These challenges are due to both cost and geographic availability. Under these conditions, other more available tests such as odor identification tests and TCS may provide a practical option and improve the accuracy of diagnosis (8).

SNH region disclosed by TCS is a marker of vulnerability to nigrostriatal dysfunction. Interestingly, approximately $8-10 \%$ of adult healthy controls in all age groups also show this feature. With the high accuracy found in our results, TCS can support a differential diagnosis and discern between PD and healthy patients. Other studies have found similar accuracy to discern PD from essential tremor, vascular parkinsonism and atypical parkinsonism $(11,12)$. TCS showed high sensitivity (91.4\%) and specificity (90\%), with a positive predictive value of $90.1 \%$ in the detection of clinical PD diagnosis (11). In our study, we observed a better sensitivity than specificity of PD diagnosis based on TCS. The differences between studies regarding accuracy of TCS with PD diagnosis may be related to inter-observer differences. TCS has a clear methodology (13), but the observer experience may explain these differences. Beyond the accuracy of PD diagnosis, $\mathrm{SNH}$ has been considered to be a risk factor for PD development. One study followed healthy subjects with SNH at baseline for 37 months. This study found an association between $\mathrm{SNH}$ and the subsequent development of a 17-times increased risk (3). A smaller research study followed 24 patients with olfactory loss, of which 11 patients had SNH. 7\% of those individuals developed PD after 4 years. None of the 14 patients with normal findings at TCS developed clinically relevant motor abnormalities (14). 
More significant olfactory impairment was observed in the PD group compared to controls, and we also found a negative correlation between the SN echogenic area and SST-16 performance. A previous study using the same method to evaluate olfaction in Brazilian PD patients revealed a specificity of $89.0 \%$ and a sensitivity of $81.1 \%$ to olfactory dysfunction diagnosed by SST-16 (8). An SST with 12 items was previously used to discriminate PD patients from controls (15). A cross-sectional study conducted by Berg et al analyzed healthy patients without PD and, as in our study, also discovered a correlation between $\mathrm{SNH}$ and lower odor identification capability (OR 1.48, 95\% CI 1.12-1.96) (16). Another study combined TCS with SST-12 and calculated the incidence of abnormal results in both tests in PD, essential tremor $(E T)$ and healthy patients (17). $63 \%$ of patients with PD were abnormal for both tests, whereas none of the patients with ET, and only 2 of the controls followed the same pattern (17). Other studies combined TCS, a test for olfactory dysfunction, and the reduced uptake of 123I-metaiodobenzylguanidine (MIBG) in myocardial scintigraphy. The combination of TCS and olfactory testing (if either one or both revealed abnormalities) discriminated patients with PD from healthy subjects with a sensitivity of $100 \%$ and $61.8 \%$, specificity of $71.9 \%$ and $100 \%$, positive predictive value of $78.6 \%$ and $100 \%$, and negative predictive value of $100 \%$ and $72.7 \%$, respectively. It was concluded that the combination of both tests substantially increased the sensitivity to a sufficient level for discriminating patients with PD from controls (18).

TRODAT SPECT has a high specificity of $98.6 \%$, and a sensitivity of $99.4 \%$ in confirming neurodegenerative parkinsonisms (19). Although the 
combination of TCS and tests to detect olfactory dysfunction has a wellestablished role to increasing PD diagnosis, few studies have compared their abnormal changes together with TRODAT SPECT findings. However, TCS alone or a smell test alone has already been compared with the accuracy of 99mTc-TRODAT-1 SPECT to PD diagnosis. In our results, we observed the absence of correlation between echogenic area of SN and TRODAT SPECT binding values and the presence of correlation between performance at SST-16 and TRODAT SPECT binding values. $\mathrm{Li}$ et al presented similar findings when comparing TCS and SPECT, and it was shown that TCS findings had no correlation with TRODAT SPECT findings. Using TRODAT SPECT as a reference method, the sensitivity and specificity of TCS to PD diagnosis were $64.7 \%$ and $60 \%$, respectively (17). Using the University of Pennsylvania Smell Identification Test, Siderowf et al analyzed olfactory impairment and SPECT binding values. They also observed a positive correlation between performance on the chosen smell test with TRODAT uptake in the striatum $(r=0.66, p=0.001)$ and putamen $(r=0.74 ; p=0.001)(20)$.

Our study performed a comparison between accuracy of abnormal results in both tests (TCS + SST-16) in comparison to abnormal results in 99mTc-TRODAT-1 SPECT alone. Bradvica et al (5) applied TCS, Pocket Smell Test and 99mTc-TRODAT-1 SPECT in PD, healthy and essential tremor patients. They observed a high correlation between transcranial sonography and DATSPECT findings (with a Kappa index=0.835), similar to our results. Sensitivity of Pocket Smell Test to PD diagnosis was $74.6 \%$, and its and specificity was $80.4 \%$ (5). However, Bradvica et al did not combine the more 
available methods to calculate their accuracy in comparison with TRODAT SPECT findings.

Previous studies performed TCS combined with tests for olfactory dysfunction and TRODAT SPECT in European populations of PD patients. This study has a clear role in the description of the validity of TCS and SPECT using DAT tracers in a Latin American PD cohort (21). However, there were some limitations: a small sample size, particularly at control group; the variation of the time span between SPECT and TCS (6 - 23 months), which likely had little influence on our results because longitudinal studies demonstrated that SN echogenic area does not change over time; and the lack of brain imaging studies in most of our patients (21).

\section{Conclusion}

We concluded that TCS and SST-16 are reliable, highly accurate methods for distinguishing patients with neurodegenerative parkinsonism from healthy controls in comparison to DATSPECT. The accuracy of TCS combined with SST-16 for differentiation between idiopathic PD patients and healthy controls is close to the that of DATSPECT performed alone as the reference method. The combined assessment of these two widely available and lower cost previous methods has a greater accuracy than each one alone, which is essential for improving the diagnosis of Parkinson's disease. 


\section{Tables}

Table 01: Demographic and clinic characteristics of the Parkinson's and control groups

\begin{tabular}{cccc}
\hline Variable & mean \pm SD & median $(25-75 \%)$ & "p" value \\
& & & \\
\hline $\begin{array}{c}\text { Gender (M/F) } \\
\text { Control group }\end{array}$ & $6 / 3$ & - & $0.930 \mathrm{a}$ \\
PD group & $13 / 7$ & & \\
& & & \\
Age (years) & & & \\
Control group & $49.77 \pm 5.04$ & $51(46 ; 54)$ & \\
$\quad$ PD group & $60.75 \pm 9.56$ & $62.5(54 ; 67.5)$ & \\
Education time in & & & \\
$\quad$ years & 13.00 & $13(10 ; 15.5)$ & \\
Control group & 5.5 & $5.5(4 ; 12)$ & - \\
$\quad$ PD group & & & - \\
Age at initial & $59.25 \pm 13.41$ & $56.5(49.0 ; 66.7)$ & - \\
symptoms & & & \\
Disease duration & $4.70 \pm 3.04$ & $5.0(2.2 ; 5.7)$ & - \\
UPDRS III & $28.60 \pm 14.57$ & $29.0(17.5 ; 35.5)$ & \\
Beck Score & & - & \\
Control group & $5.77 \pm 3.27$ & & \\
PD group & $10.30 \pm 3.82$ & & \\
MMEE Score & & & \\
Control group & $27.66 \pm 1.80$ & & \\
PD group & $26.00 \pm 3.59$ & & \\
\hline
\end{tabular}

$a=$ Likelihood ratio test; $b=$ Mann-Whitney $U$ test; $c=$ Student's $T$ test, * Nonparametric variable $=$ values expressed by median . 
Table 02: Description of values obtained from transcranial sonography, TRODAT and performance on the hyposmia test in the Parkinson's and control groups

\begin{tabular}{|c|c|c|c|c|c|c|c|}
\hline \multirow[t]{2}{*}{ Variable } & \multicolumn{3}{|c|}{ Control Group } & \multicolumn{3}{|c|}{ Parkinson's group } & \multirow[t]{2}{*}{ "p" value* } \\
\hline & 25 & Median & 75 & 25 & Median & 75 & \\
\hline $\begin{array}{l}\text { SN echogenicity } \\
\text { area }(\mathrm{cm} 2)\end{array}$ & 0.14 & 0.17 & 0.43 & 0.40 & 0.51 & 0.65 & 0.013 \\
\hline $\begin{array}{l}\text { Striatal 99mTc- } \\
\text { TRODAT uptake }\end{array}$ & 1.03 & 1.18 & 1.41 & 0.47 & 0.58 & 0.67 & 0.001 \\
\hline $\begin{array}{l}\text { Performance on the } \\
\text { hyposmia test }\end{array}$ & 11.00 & 12.00 & 13.00 & 5.25 & 9.00 & 10.7 & 0.001 \\
\hline
\end{tabular}

* Mann-Whitney U test 
Table 03: Cut-off values obtained from transcranial sonography, TRODAT and performance on the hyposmia test to Parkinson's disease diagnosis

\begin{tabular}{lllccc}
\hline Variable & & \multicolumn{4}{c}{ Group } \\
& $\begin{array}{l}\text { Cutoff } \\
\text { points }\end{array}$ & & Control & PD & $\begin{array}{c}\text { "p" } \\
\text { value* }\end{array}$ \\
\hline SN echogenicity & $\geq 0.22$ & $N$ & 2 & 20 & \\
area (cm2) & & $\%$ & 22.2 & 100.0 & 0.001 \\
& $<0.22$ & $N$ & 7 & 0 & \\
Striatal 99mTc- & $\geq 0.90$ & $N$ & 8 & 0 & \\
TRODAT uptake & & $\%$ & 88.9 & 0 & 0.001 \\
& $<0.90$ & $N$ & 1 & 20 & \\
Performance on & $\geq 10$ & $N$ & 11.1 & 100 & \\
the hyposmia test & & $\%$ & 9 & 7 & \\
& $<10$ & $N$ & 100 & 35 & 0.001 \\
& & $\%$ & 0 & 13 & \\
\hline
\end{tabular}

* The Chi-square test, PD= Parkinson's group 
Table 04: Accuracy of transcranial sonography, TRODAT, hyposmia test and combined hyposmia test plus transcranial sonography to Parkinson's disease diagnosis

\begin{tabular}{lcccc}
\hline \multicolumn{1}{c}{ Variable } & Area & IC $<95 \%$ & IC>95\% & "P" value \\
\hline $\begin{array}{l}\text { SN echogenicity } \\
\text { area (cm2) }\end{array}$ & 0.792 & 0.536 & 1.001 & 0.013 \\
$\begin{array}{l}\text { Performance on the } \\
\text { hyposmia test }\end{array}$ & 0.858 & 0.725 & 0.992 & 0.002 \\
$\begin{array}{l}\text { Striatal 99mTc- } \\
\text { TRODAT uptake }\end{array}$ & 0.998 & 0.982 & 1.001 & 0.001 \\
$\begin{array}{l}\text { Performance on the } \\
\text { hyposmia test }+\end{array}$ & 0.95 & 0.869 & 1.000 & 0.001 \\
$\begin{array}{l}\text { SN echogenicity } \\
\text { area (cm2) }\end{array}$ & & & & \\
\hline
\end{tabular}




\section{References}

1. Doepp F, Plotkin M, Siegel L, Kivi A, Gruber D, Lobsien E, Kupsch A, Schreiber SJ. Brain parenchyma sonography and 123I-FP-CIT SPECT in Parkinson's disease and essential tremor. Mov Disord. 2008: 23(3):405-10.

2. Berg D, Siefker C, Becker G. Echogenicity of the substantia nigra in Parkinson's disease and its relation to clinical findings. J Neurol. 2001; 248:684- 689.

3. Berg D, Seppi K, Behnke S, Liepelt I, Schweitzer K, Stockner H, Wollenweber F, Gaenslen A, Mahlknecht P, Spiegel J, Godau J, Huber H, Srulijes K, Kiechl S, Bentele M, Gasperi A, Schubert T, Hiry T, Probst M, Schneider V, Klenk J, Sawires M, Willeit J, Maetzler W, Fassbender K, Gasser T, Poewe W. Enlarged substantia nigra hyperechogenicity and risk for Parkinson disease: a 37-month 3-center study of 1847 older persons. Arch Neurol. 2011; 68: 932- 937.

4. Berg D. Substantia nigra hyperechogenicity is a risk marker of Parkinson's disease: yes. J Neural Transm. 2011;118(4):613-9. 
5. Štenc Bradvica I, Mihaljević I, Butković-Soldo S, Kadojić D, Titlić M, Bradvica M, Kralik K. Transcranial sonography and the pocket smell test in the differential diagnosis between parkinson's disease and essential tremor. Neurol Sci. 2015; 36(8):1403-10.

6. Brucki SM, Nitrini R, Caramelli P, Bertolucci PH, Okamoto IH. [Suggestions for utilization of the mini-mental state examination in Brazil]. Arq Neuropsiquiatr. 2003; 61:777- 781.

7. Bor-Seng-Shu E, Felicio AC, Braga-Neto P, Batista IR, Paiva WS, de Andrade DC, Teixeira MJ, de Andrade LA, Barsottini OG, Shih MC, Bressan RA, Ferraz HB. Dopamine transporter imaging using 99mTc-TRODAT-1 SPECT in Parkinson's disease. Med Sci Monit. 2014; 20:1413-8.

8. Silveira-Moriyama L, Carvalho MJ, Katzenschlager R, Petrie A, Ranvaud R, Barbosa ER, Lees AJ. The use of smell identification tests in the diagnosis of Parkinson's disease in Brazil. Mov Disord. 2008; 23:2328-2334.

9. Santin R, Fonseca VF, Bleil CB, Rieder CR, Hilbig A. Olfactory function and Parkinson's disease in Southern Brazil. Arq Neuropsiquiatr. 2010; 68(2): 252-7. 
10. Barbosa MT, Caramelli P, Maia DP, Cunningham MC, Guerra HL, LimaCosta MF, Cardoso F. Parkinsonism and Parkinson's disease in the elderly: a community-based survey in Brazil (the Bambui study). Mov Disord. 2006; 21:800- 808 .

11. Budisic M, Trkanjec Z, Bosnjak J, Lovrencic-Huzjan A, Vukovic V, Demarin V. Distinguishing Parkinson's disease and essential tremor with transcranial sonography. Acta Neurol Scand. 2009; 119(1): 17-2.

12. Walter U, Dressler D, Probst T, Wolters A, Abu-Mugheisib M, Wittstock M, Benecke R. Transcranial brain sonography findings in discriminating between parkinsonism and idiopathic Parkinson disease. Arch Neurol. 2007; 64(11): 1635-40.

13. Walter U, Behnke S, Eyding J, Niehaus L, Postert T, Seidel G, Berg D. Transcranial brain parenchyma sonography in movement disorders: state of the art. Ultrasound Med Biol. 2007; 33 (1):15-25.

14. Haehner A, Hummel T, Hummel C, Sommer U, Junghanns S, Reichmann H. Olfactory loss may be a first sign of idiopathic Parkinson's disease. Mov Disord. 2007; 22(6): 839-42. 
15. Busse K, Heilmann R, Kleinschmidt S, Abu-Mugheisib M, Höppner J, Wunderlich C, Gemende I, Kaulitz L, Wolters A, Benecke R, Walter U. Value of combined midbrain sonography, olfactory and motor function assessment in the differential diagnosis of early Parkinson's disease. J Neurol Neurosurg Psychiatry. 2012; 83(4): 441-7.

16. Berg D, Seppi K, Liepelt I, Schweitzer K, Wollenweber F, Wolf B, Dillmann U, Stockner H, Godau J, Kiechl S, Gaenslen A, Willeit J, Di Santo A, Maetzler W, Gasser T, Poewe W, Behnke S. Enlarged hyperechogenic substantia nigra is related to motor performance and olfaction in the elderly. Mov Disord. 2010; 25(10): 1464-9.

17. Li DH, Zhang LY, Hu YY, Jiang XF, Zhou HY, Yang Q, Kang WY, Liu J, Chen SD. Transcranial sonography of the substantia nigra and its correlation with DAT-SPECT in the diagnosis of Parkinson's disease. Parkinsonism Relat Disord. 2015; 21(8): 923-8.

18. Izawa MO, Miwa $\mathrm{H}$, Kajimoto $\mathrm{Y}$, Kondo $\mathrm{T}$. Combination of transcranial sonography, olfactory testing, and MIBG myocardial scintigraphy as a diagnostic indicator for Parkinson's disease. Eur J Neurol. 2012;19(3): 411-6. 
19. Bajaj N, Hauser RA, Grachev ID. Clinical utility of dopamine transporter single photon emission CT (DaT-SPECT) with (123I) ioflupane in diagnosis of parkinsonian syndromes. J Neurol Neurosurg Psychiatry 2013; 11:1288 1295.

20. Siderowf A, Newberg A, Chou KL, Lloyd M, Colcher A, Hurtig HI, Stern MB, Doty RL, Mozley PD, Wintering N, Duda JE, Weintraub D, Moberg PJ. [99mTc] TRODAT-1 SPECT imaging correlates with odor identification in early Parkinson disease. Neurology. 2005; 64(10):1716-20.

21. Bor-Seng-Shu E, Pedroso JL, Felicio AC, de Andrade DC, Teixeira MJ, Braga-Neto P, Batista IR, Barsottini OG, Borges V, Ferraz HB, Shih MC, Bressan RA, de Andrade LA, Walter U. Substantia nigra echogenicity and imaging of striatal dopamine transporters in Parkinson's disease: a crosssectional study. Parkinsonism Relat Disord. 2014; 20(5):477-81. 